\title{
The arithmetic of elliptic fibrations in gauge theories on a circle
}

\author{
Thomas W. Grimm, ${ }^{a, b, c}$ Andreas Kapfer ${ }^{a}$ and Denis Klevers ${ }^{d}$ \\ ${ }^{a}$ Max-Planck-Institut für Physik, \\ Föhringer Ring 6, 80805 Munich, Germany \\ ${ }^{b}$ Institute for Theoretical Physics, \\ Utrecht University, Leuvenlaan 4, 3584 CE Utrecht, The Netherlands \\ ${ }^{c}$ Center for Extreme Matter and Emergent Phenomena, \\ Utrecht University, Leuvenlaan 4, 3584 CE Utrecht, The Netherlands \\ ${ }^{d}$ Theory Group, Physics Department, CERN, \\ CH-1211, Geneva 23, Switzerland \\ E-mail: grimm@mpp.mpg.de, kapfer@mpp.mpg.de, denis.klevers@cern.ch
}

ABSTRACT: The geometry of elliptic fibrations translates to the physics of gauge theories in F-theory. We systematically develop the dictionary between arithmetic structures on elliptic curves as well as desingularized elliptic fibrations and symmetries of gauge theories on a circle. We show that the Mordell-Weil group law matches integral large gauge transformations around the circle in Abelian gauge theories and explain the significance of Mordell-Weil torsion in this context. We also use Higgs transitions and circle large gauge transformations to introduce a group law for genus-one fibrations with multi-sections. Finally, we introduce a novel arithmetic structure on elliptic fibrations with non-Abelian gauge groups in F-theory. It is defined on the set of exceptional divisors resolving the singularities and divisor classes of sections of the fibration. This group structure can be matched with certain integral non-Abelian large gauge transformations around the circle when studying the theory on the lower-dimensional Coulomb branch. Its existence is required by consistency with Higgs transitions from the non-Abelian theory to its Abelian phases in which it becomes the Mordell-Weil group. This hints towards the existence of a new underlying geometric symmetry.

KEYwords: Effective field theories, F-Theory, Gauge Symmetry, M-Theory

ArXiv EPrint: 1510.04281 


\section{Contents}

1 Introduction 1

2 Symmetries of gauge theories on the circle 5

2.1 General setup and circle reduction 5

2.2 Large gauge transformations on the Coulomb branch 8

3 On the systematics of F-theory compactifications 11

4 Arithmetic structure on fibrations with rational sections $\quad \mathbf{1 5}$

$\begin{array}{lll}4.1 & \text { On the Mordell-Weil group and its divisor group law } & 15\end{array}$

$\begin{array}{ll}4.2 & \text { The free part of the Mordell-Weil group } \\ & 16\end{array}$

$\begin{array}{lll}\text { 4.3 The torsion part of the Mordell-Weil group } & 18\end{array}$

5 Introducing an arithmetic structure on fibrations with multi-sections $\quad 20$

5.1 A group action for fibrations with multi-sections 21

$\begin{array}{ll}5.2 & \text { The generalized Shioda map } \\ 5.3\end{array}$

5.3 Extended Mordell-Weil group and large gauge transformations 27

6 Introducing an arithmetic structure on fibrations with exceptional $\begin{array}{ll}\text { divisors } & \mathbf{2 8}\end{array}$

$\begin{array}{lll}6.1 & \text { A group action for exceptional divisors } & 29\end{array}$

6.2 Arithmetic group structures from Higgs transitions 32

$\begin{array}{lll}7 & \text { Conclusions } & 34\end{array}$

A Lie theory conventions $\quad 36$

\section{Introduction}

In recent years the connection of the geometry of elliptic curves to gauge theories in various dimensions has been explored intensively by using F-theory [1]. In F-theory two auxiliary dimensions need to be placed on a two-torus whose complex structure is identified with the Type IIB dilaton-axion. Its variations are then encoded by the geometry of a two-torus fibration in F-theory. Magnetic sources for the dilaton-axion are seven-branes that support gauge theories. Several features of these gauge theories can thus be studied using two-torus fibrations. At first, the F-theory approach seems to suggest that the connection between geometry and gauge theories is rather direct. However, it turns out that the geometry of elliptic fibrations should rather be related to gauge theories compactified on a circle. This can be understood by realizing that the volume of the two-torus is unphysical in F-theory 
and that there is no notion of an actual twelve-dimensional background geometry. The geometry of the elliptic fibration of F-theory is only fully probed in the dual M-theory compactification. M-theory compactified on an elliptic fibration yields the effective theory of F-theory compactified on an additional circle. In particular, one is therefore forced to relate the geometry of elliptic fibrations with gauge theories on a circle. In this work we will further develop the dictionary between the geometry of elliptic fibrations and mattercoupled gauge theories on a circle. Our focus here will be on revealing geometric symmetry transformations that correspond to large gauge transformations around the circle.

As a first example of such a relation we will study Abelian gauge theories on a circle. In an F-theory compactification the number of massless U(1) fields can be related to the number of rational sections or multi-sections minus one [2]. The section that is not counted here has to be identified with the Kaluza-Klein vector obtained from the higher-dimensional metric when placing the gauge theory on the additional circle. Recent progress in understanding $\mathrm{U}(1)$ gauge groups in F-theory can be found, for example, in the references [3-17]. The fact that smooth geometries carry information about a circle reduced theory becomes particularly apparent in models with rational sections in which the mass hierarchy between Kaluza-Klein masses and lower-dimensional Coulomb branch masses is non-trivial [9]. In other words, it was key in $[9,18]$ that despite the fact that massive states have to be integrated out in the circle compactified effective theory some cutoff independent information about the massive tower has to be kept. In particular, the one-loop Chern-Simons terms carry information about the representations of the higherdimensional chiral spectrum supplemented with a table of signs for each state $[19,20]$. This extra information can be summarized in so-called box graphs introduced in [21, 22], see also [23, 24]. In general, however, it is important to also keep track of an integer label for each dimensionally reduced state that encodes the mass hierarchy between the Kaluza-Klein mass and the Coulomb branch mass. For models with only a multi-section, see [15, 25-29] for representative works, the requirement of a lower-dimensional approach is even more pressing. As discussed in [28, 30-33] the multi-section should be understood as a mixing of the higher-dimensional U(1)s and the Kaluza-Klein vector.

The first goal of this work is to formalize the relationship between geometries with rational sections and circle reduced gauge theories further. We carefully identify the MordellWeil group acting on rational sections as large gauge transformations along the circle, as already suggested in [18]. The Mordell-Weil group is a discrete finitely-generated Abelian group that captures key information about the arithmetic of elliptic fibrations. We show that there is indeed a one-to-one correspondence of large gauge transformations with generators of the Mordell-Weil group. The free part of the Mordell-Weil group is identified with Abelian large gauge transformations, while its torsion part is related to special fractional non-Abelian large gauge transformations. As a byproduct we explore the geometric relationship between the existence of fractional Abelian charges of matter states and the presence of a non-Abelian gauge group.

A second goal of this work is to use our understanding of the arithmetic for geometries with rational sections to provide evidence for the existence of a natural group law acting on fibrations with multi-sections. We call this group extended Mordell-Weil group, despite 
the fact that there is formally no Mordell-Weil group for multi-sections. We also define a generalized Shioda map that allows to explore the physical implications of the group action. Furthermore, we rigorously establish the correspondence of the proposed group action on the divisor level with large gauge transformations around the circle. In many examples it is known that there exist geometric transitions from a model with several sections to a model with multi-section [15, 27, 28, 30-33]. Physically this corresponds to a Higgsing of charged matter states. By construction the group law of the extended Mordell-Weil group should be inherited from the setup with multiple sections. Accordingly, it trivially reduces to the standard Mordell-Weil group law in the presence of sections only. As first explained in [18], in geometries with multiple sections the fact that one is free to choose a zero-section in the geometry to perform the F-theory limit is deeply linked to anomaly freedom of the higher-dimensional Abelian gauge theory. Using the extended Mordell-Weil group one would be able to extend these arguments to this larger class of geometries and their corresponding effective theories.

The third goal of this paper it to extend the discussion to fully include matter-coupled non-Abelian gauge theories with gauge group $G$. Placing these theories on a circle we perform large gauge transformations along the circle and explore the associated arithmetic structure in the geometry. More precisely, we are interested in examining the impact of non-Abelian large gauge transformation on the $\mathrm{U}(1)^{r+1}$ gauge theory obtained in the lower-dimensional Coulomb branch. Here $r$ is the rank of $G$ and the additional $\mathrm{U}(1)$ is the Kaluza-Klein vector of the higher-dimensional metric. The circle gauge transformations mix these vectors non-trivially and it was argued in [18] that the identification of the effective theories is only possible if non-Abelian anomalies are cancelled. However, the group structure encoding the allowed changes remained mysterious in [18]. In this work we show that there indeed is a natural group structure on the set of exceptional divisors and rational sections corresponding to these large gauge transformations. We also make progress in identifying the geometric symmetry corresponding to such transformations. First, we show that the transformed exceptional divisors and rational sections can have a standard geometry interpretation. Second, we employ that a non-Abelian gauge theory with adjoints is related to an Abelian theory with $r \mathrm{U}(1) \mathrm{s}$ by Higgsing/unHiggsing corresponding to complex structure deformations in the geometry. Using recent results in $[17,27]$ we show that the postulated group structure on the exceptional divisors gets mapped precisely to the usual Mordell-Weil group law of the geometry corresponding to the Abelian theory. All this hints at the existence of a geometrical symmetry directly in the geometry associated to the non-Abelian theory that is yet to be formulated explicitly. In fact, on the field theory level this is clear since the circle-compactified theories only differ by a non-Abelian large gauge transformation. This is obviously a symmetry of an anomaly free gauge theory, which indicates that the corresponding geometry should be considered physically equivalent to the original elliptic fibration.

Before starting with the actual computations, let us note that our intuition can be summarized by using figure 1 as follows. First, we are able to establish the relation of the Mordell-Weil group to large Abelian gauge transformations in elliptic fibrations with rational sections depicted in (A). Using a geometric transition, which corresponds to Higgsing 


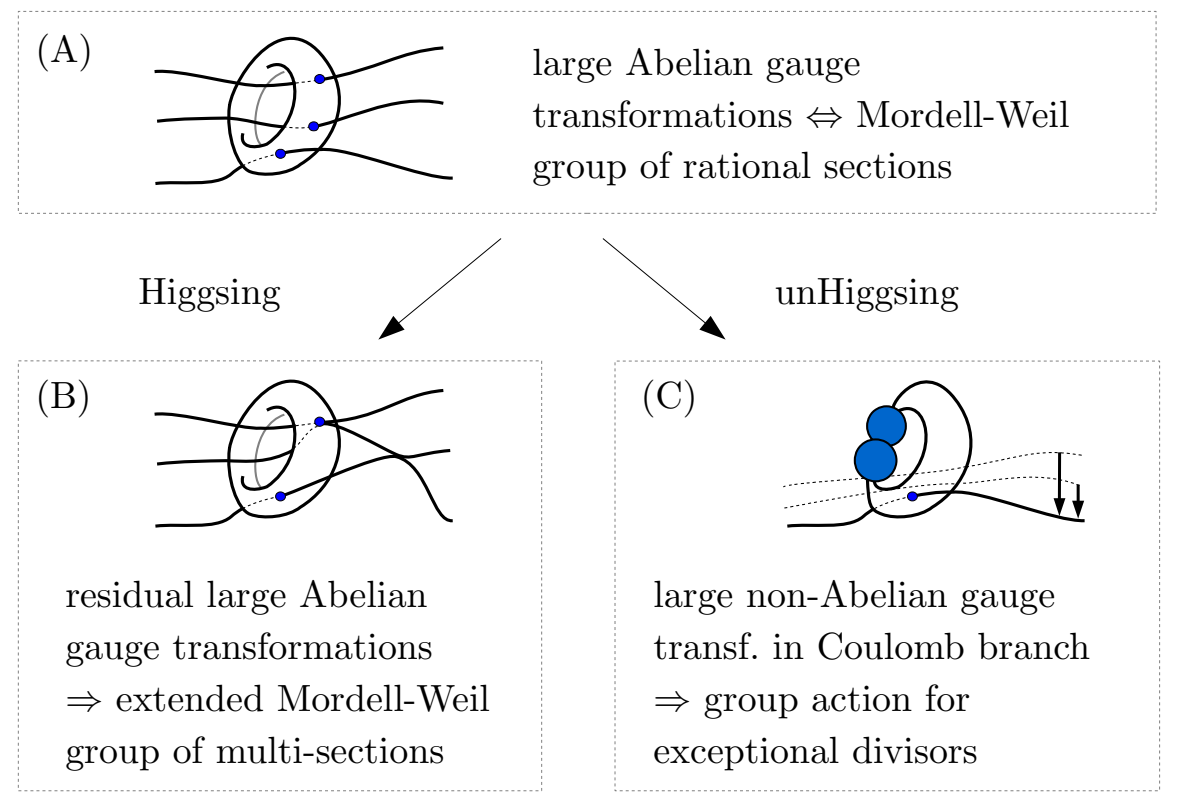

Figure 1. Schematic depiction of the various geometric configurations considered in this work. The geometries are related by geometric transitions describing Higgsing and unHiggsing processes.

in field theory, the resulting fibrations might only admit multi-sections, see (B). Therefore, one expects an extended Mordell-Weil group structure for such geometries. Furthermore, a geometry with rational sections might arise via a geometric transition describing a nonAbelian gauge theory. Again this is described by a Higgsing in field theory. Such transitions motivate us to transfer the Mordell-Weil group structure to a geometry with exceptional divisors. The resulting group structure corresponds to large non-Abelian gauge transformation for an F-theory effective field theory compactified on a circle and pushed to the Coulomb branch.

The paper is organized as follows. In section 2 we discuss the compactification of Abelian and non-Abelian gauge theories on a circle. We introduce our notation and the relevant large gauge transformations along the circle. The treatment will be valid both for six- and four-dimensional gauge theories. We also briefly comment on anomalies and one-loop Chern-Simons terms induced after circle compactification. A summary of some basic facts about F-theory compactifications and elliptic fibrations is provided in section 3. In section 4 we focus on geometries with rational sections and the corresponding Abelian parts of the gauge theory. The Mordell-Weil group action is mapped to large gauge transformations along the circle. We also discuss the impact of torsion from this perspective. In section 5 we then turn to geometries with multi-sections. Insights obtained by using Higgs transitions allow us to define an extended Mordell-Weil group of multi-sections and a generalized Shioda map. In section 6 we extend the analysis further to cover non-Abelian gauge groups. We argue for the existence of a group law on the exceptional divisors and rational sections that is shown to be induced by large gauge transformations of the Cartan 
gauge fields. We geometrically motivate its existence by explicitly considering Higgsings to Abelian gauge theories.

\section{Symmetries of gauge theories on the circle}

In this section we discuss a number of symmetries that are encountered when considering six- and four-dimensional gauge theories on a circle. In subsection 2.1 we first introduce our notation and summarize some basic facts about circle compactifications of gauge theories. In subsection 2.2 we study the manifestation of large gauge transformations with gauge parameters supported on the circle. We comment on the rearrangement of the Kaluza-Klein spectrum and the significance of one-loop induced Chern-Simons terms on the Coulomb branch of the gauge theory.

\subsection{General setup and circle reduction}

We begin by introducing some general notions about Abelian and non-Abelian gauge theories in six and four space-time dimensions. Let us denote by $G$ a simple gauge group with gauge bosons $\hat{A}$. Introducing the Lie algebra generators $T_{\mathcal{I}}, \mathcal{I}=1, \ldots, \operatorname{dim} G$ we expand

$$
\hat{A}=\hat{A}^{\mathcal{I}} T_{\mathcal{I}}=\hat{A}^{I} T_{I}+\hat{A}^{\boldsymbol{\alpha}} T_{\boldsymbol{\alpha}}
$$

where $T_{I}, I=1, \ldots, \operatorname{rank} G$ are the generators of the Cartan subalgebra and $T_{\boldsymbol{\alpha}}$ are the remaining generators labeled by the roots $\boldsymbol{\alpha}$. In addition, we will allow for a number $n_{\mathrm{U}(1)}$ of Abelian gauge bosons that are denoted by $\hat{A}^{m}$ with $m=1, \ldots, n_{\mathrm{U}(1)}$.

In the next step we compactify this theory on a circle and push it to the Coulomb branch by allowing for a non-vanishing Wilson line background of the gauge fields reduced on the circle. From the four- or six-dimensional metric one finds at lowest level the threeor five-dimensional metric $g_{\mu \nu}$, the Kaluza-Klein vector $A^{0}$, and the radius $r$ of the circle. Thus, the higher-dimensional line element is expanded as

$$
d \hat{s}^{2}=g_{\mu \nu} d x^{\mu} d x^{\nu}+r^{2} D y^{2}, \quad D y:=d y-A_{\mu}^{0} d x^{\mu},
$$

with $x^{\mu}$ the three- or five-dimensional coordinates and $y$ the coordinate along the circle. Performing the Kaluza-Klein reduction of the vector fields one finds at lowest Kaluza-Klein level a number of $\operatorname{dim} G$ gauge fields $A^{\mathcal{I}}$ and $\operatorname{dim} G$ Wilson line scalars $\zeta^{\mathcal{I}}$ from reducing $\hat{A}$. In addition one has $n_{\mathrm{U}(1)} \mathrm{U}(1)$ gauge fields $A^{m}$ and Wilson line scalars $\zeta^{m}$ from reducing $\hat{A}^{m}$. In particular the gauge fields are expanded as

$$
\hat{A}^{\mathcal{I}}=A^{\mathcal{I}}-\zeta^{\mathcal{I}} r D y, \quad \hat{A}^{m}=A^{m}-\zeta^{m} r D y .
$$

The $A^{\mathcal{I}}$ are now gauge fields of the lower-dimensional version of the gauge group $G$, while the $\zeta^{\mathcal{I}}$ transform in the adjoint representation of $G$. In other words, denoting the gauge parameters by $\Lambda^{\mathcal{I}}(x)$ and $\Lambda^{m}(x)$, one has

$$
\begin{aligned}
& \delta A^{\mathcal{I}}=d \Lambda^{\mathcal{I}}+f_{\mathcal{J} \mathcal{K}}^{\mathcal{I}} \Lambda^{\mathcal{J}} A^{\mathcal{K}}, \\
& \delta \zeta^{\mathcal{I}}=f_{\mathcal{J} \mathcal{K}}^{\mathcal{I}} \zeta^{\mathcal{J}} \Lambda^{\mathcal{K}}, \\
& \delta A^{m}=d \Lambda^{m}, \\
& \delta \zeta^{m}=0 \text {, }
\end{aligned}
$$


where $f_{\mathcal{J K}}^{\mathcal{I}}$ are the structure constants of $G$. For this work it is crucial to realize that there is whole class of higher-dimensional gauge transformations, with gauge parameters $\hat{\Lambda}^{\mathcal{I}}(x, y)$ and $\hat{\Lambda}^{m}(x, y)$ depending non-trivially on $y$, which are not included in (2.4). We will discuss these additional transformations in subsection 2.2 in more detail.

The Coulomb branch of the compactified theory is parametrized by the background values of the scalars $\zeta^{\mathcal{I}}$ and $\zeta^{m}$, by setting

$$
\left\langle\zeta^{I}\right\rangle \neq 0, \quad\left\langle\zeta^{\boldsymbol{\alpha}}\right\rangle=0, \quad\left\langle\zeta^{m}\right\rangle \neq 0
$$

i.e. giving the Cartan Wilson line scalars a vacuum expectation value. This induces the breaking

$$
G \times \mathrm{U}(1)^{n_{\mathrm{U}(1)}} \rightarrow \mathrm{U}(1)^{\mathrm{rank} G} \times \mathrm{U}(1)^{n_{\mathrm{U}(1)}},
$$

and assigns a mass to the W-bosons $A^{\alpha}$. Note that one has to include in addition the Kaluza-Klein vector $A^{0}$, such that the full three- or five-dimensional massless gauge group is actually $\mathrm{U}(1)^{\operatorname{rank} G+n_{\mathrm{U}(1)}+1}$.

The massive fields in the lower-dimensional theory are then the excited Kaluza-Klein modes of all higher-dimensional states and the fields that acquire masses on the Coulomb branch. In particular, also the modes of the higher-dimensional charged matter states will gain a mass. Let us, for example, consider a fermion $\hat{\psi}$ in the higher-dimensional theory that transforms in some representation $R$ under $G$ and has U(1)-charges $q_{m}$ under the gauge fields $\hat{A}^{m}$. This implies that the higher-dimensional covariant derivative takes the form

$$
\mathcal{D}_{\mu} \hat{\psi}=\left(\nabla_{\mu} \hat{\psi}-i \hat{A}_{\mu}^{\mathcal{I}} T_{\mathcal{I}}^{R}-i q_{m} \hat{A}_{\mu}^{m}\right) \hat{\psi}
$$

We expand $\hat{\psi}$ in an eigenbasis $\hat{\psi}(w)$ associated to the weights $w$ of $R$ with the property $T_{I}^{R} \hat{\psi}(w)=w_{I} \hat{\psi}(w)$ for the Cartan directions, where $w_{I}:=\left\langle\boldsymbol{\alpha}_{I}^{\vee}, w\right\rangle$ are the Dynkin labels and $\boldsymbol{\alpha}_{I}^{\vee}$ is the simple coroot associated to $T_{I}$. We refer to appendix A for our conventions for Lie groups. Dimensionally reducing such a fermion $\hat{\psi}(w, q)$ on a circle, one can infer its Coulomb branch mass $m_{\mathrm{CB}}^{w, q}$ in the background (2.5). Using (2.7) we read off

$$
m_{\mathrm{CB}}^{w, q}=w_{I}\left\langle\zeta^{I}\right\rangle+q_{m}\left\langle\zeta^{m}\right\rangle
$$

In total the mass of a field $\psi_{(n)}(w, q)$ at Kaluza-Klein level $n$ in the lower-dimensional theory reads

$$
m=m_{\mathrm{CB}}^{w, q}+n m_{\mathrm{KK}}=w_{I}\left\langle\zeta^{I}\right\rangle+q_{m}\left\langle\zeta^{m}\right\rangle+\frac{n}{\langle r\rangle},
$$

with $m_{\mathrm{KK}}=1 /\langle r\rangle$ being the unit Kaluza-Klein mass determined by the background value of the radius. Note that a similar analysis can be performed for the Kaluza-Klein modes of all other fields, including scalars, W-bosons, and six-dimensional tensor fields.

In particular, let us denote by $T_{\mathrm{sd}}$ and $T_{\text {asd }}$ the number of six-dimensional self-dual and anti-self-dual tensors respectively. Each such tensor $\hat{B}^{\alpha}, \alpha=1, \ldots, T_{\mathrm{sd}}+T_{\text {asd }}$, yields a whole tower of Kaluza-Klein states after compactification on the circle. While the massive modes are genuine tensor fields in five dimensions [34, 35], the massless mode can be dualized into 
a massless five-dimensional vector field $A^{\alpha} \cdot{ }^{1}$ To be more precise, the Kaluza-Klein ansatz for $\hat{B}^{\alpha}$ reads

$$
\hat{B}^{\alpha}=B^{\alpha}-\left(A^{\alpha}-2 \lambda_{G}^{-1} b^{\alpha} \operatorname{tr}_{\mathrm{f}}(\zeta A)-2 b_{m n}^{\alpha} \zeta^{m} A^{n}\right) \wedge D y .
$$

The group-specific coefficient is given by $\lambda_{G}^{-1}=\left\langle\boldsymbol{\alpha}_{\max }, \boldsymbol{\alpha}_{\max }\right\rangle / 2$, where $\boldsymbol{\alpha}_{\max }$ is the root of maximal length (see appendix A). The trace $\operatorname{tr}_{f}$ is evaluated in the fundamental representation of $G$. Note that the modification of this ansatz with terms proportional to the constant Green-Schwarz coefficients $b^{\alpha}$ and $b_{m n}^{\alpha}$, to be defined below in (2.12), is important since the six-dimensional tensors have modified field strengths (see e.g. [9, 36] for a more complete discussion). They therefore can participate non-trivially in the Green-Schwarz mechanism as we discuss momentarily. In the classical five-dimensional Coulomb branch parametrized by (2.5) the ansatz (2.10) including only the massless fields becomes

$$
\hat{B}^{\alpha}=B^{\alpha}-\left(A^{\alpha}-2 b^{\alpha} \mathcal{C}_{I J} \zeta^{I} A^{J}-2 b_{m n}^{\alpha} \zeta^{m} A^{n}\right) \wedge D y,
$$

where we have introduced the coroot intersection matrix $\mathcal{C}_{I J}=\lambda_{G}^{-1} \operatorname{tr}_{\mathrm{f}}\left(T_{I} T_{J}\right)$ with $T_{I}$ being the Cartan generators in the coroot basis. Again, we refer to appendix A, in particular (A.3) and the following text for more details. In five dimensions the $B^{\alpha}$ can then be eliminated from the action in favour of $A^{\alpha}$ by using the self- or anti-self-duality of $\hat{B}^{\alpha}$.

It will be important in the following that a classical four-dimensional or six-dimensional gauge theory does not necessarily have to be gauge invariant. In fact, it is well-known that often classical gauge non-invariance is required to cancel one-loop gauge anomalies induced by chiral fields. Famously, this is done by the Green-Schwarz mechanism [37-39]. In six dimensions the relevant terms are given $b y^{2}$

$$
\hat{S}_{\mathrm{GS}}^{(6)}=-\int \eta_{\alpha \beta} \hat{B}^{\beta} \wedge\left(b^{\alpha} \lambda_{G}^{-1} \operatorname{tr}_{\mathrm{f}}(\hat{F} \wedge \hat{F})+b_{m n}^{\alpha} \hat{F}^{m} \wedge \hat{F}^{n}\right),
$$

where $b^{\alpha}$ and $b_{m n}^{\alpha}$ are the Green-Schwarz anomaly coefficients. The matrix $\eta_{\alpha \beta}$ is constant, symmetric in its indices and its signature consists of $T_{\text {sd }}$ positive signs and $T_{\text {asd }}$ negative ones. Anomaly cancellation takes place if the tree-level diagram involving the tensors $\hat{B}^{\alpha}$ cancels the non-vanishing contribution of all anomalous one-loop diagrams induced by chiral fields of the theory. In six dimensions chiral fields are spin- $1 / 2$ and spin- $3 / 2$ fermions as well as self-dual or anti-self-dual tensors.

In four space-time dimensions the Green-Schwarz mechanism can be mediated by axions $\hat{\rho}_{\alpha}, \alpha=1, \ldots, n_{\mathrm{ax}}$, rather than tensor fields. The relevant classical counter-term is then given by

$$
\hat{S}_{\mathrm{GS}}^{(4)}=-\frac{1}{4} \int \eta_{\alpha}{ }^{\beta} \hat{\rho}_{\beta}\left(b^{\alpha} \lambda_{G}^{-1} \operatorname{tr}_{\mathrm{f}}(\hat{F} \wedge \hat{F})+b_{m n}^{\alpha} \hat{F}^{m} \wedge \hat{F}^{n}\right)
$$

where $\eta_{\alpha}{ }^{\beta}$ is a constant square matrix. This term is non-gauge-invariant if the axions $\hat{\rho}_{\alpha}$ are shift-gauged by the $\mathrm{U}(1)$ gauge fields $\hat{A}^{m}$. In analogy to six dimensions anomaly

\footnotetext{
${ }^{1}$ Note that the index $\alpha$ counts the number of massless tensor fields in six dimensions and should not be confused with $\boldsymbol{\alpha}$ labelling the roots of $G$.

${ }^{2}$ In general there exist also gravitational Green-Schwarz terms in order to cancel gravitational anomalies. However, these are of no importance in our discussion, and we omit them in the following.
} 
cancellation takes place if the tree-level diagram involving the axions $\hat{\rho}_{\alpha}$ cancels the nonvanishing contribution of all anomalous one-loop diagrams induced by chiral fields of the theory. In four dimensions such chiral fields are spin- $1 / 2$ fermions. It is important to point out that after compactification to three space-time dimensions the Kaluza-Klein zero-modes of the axions $\hat{\rho}_{\alpha}$ can be dualized into three-dimensional vectors $A^{\alpha}$ (see e.g. [20, 40] for a detailed discussion).

We thus realize that both the six-dimensional and four-dimensional setting is characterized by Green-Schwarz coefficients $b^{\alpha}, b_{m n}^{\alpha}$. Furthermore, the fields $\hat{B}^{\alpha}$ and $\hat{\rho}_{\alpha}$ appearing in (2.12) and (2.13) are both captured by vectors $A^{\alpha}$ in the five- and three-dimensional effective theories respectively. This slight abuse of notation will allow us to consider both the six-dimensional and four-dimensional case simultaneously. Let us also stress that all following considerations also apply to situations where no Green-Schwarz mechanism is employed to cancel anomalies.

\subsection{Large gauge transformations on the Coulomb branch}

In this subsection we discuss in detail the set of gauge transformations of an Abelian or non-Abelian theory on a circle that are translated to a symmetry of the geometry of an elliptic fibration. Recall that after compactification the effective theory admits (2.4) as local symmetries before pushed to the Coulomb branch. In the Coulomb branch one simply has a purely Abelian local symmetry.

In addition to the lower-dimensional gauge transformations (2.4) we could also have performed a circle-dependent gauge transformation and then compactified on the circle $y \sim y+2 \pi$. If one preserves the boundary conditions of the fields in the compactification ansatz the gauge invariance of the higher-dimensional theory then implies that there exists a variety of equivalent lower-dimensional effective theories that are obtained after circle reduction of the same higher-dimensional theory. Gauge transformations that cannot be deformed continuously to the identity map are known as large gauge transformations. More concretely, let us consider the effect of a gauge transformation that locally takes the form

$$
\hat{\Lambda}^{\mathcal{I}}(x, y)=\left\{\begin{array}{c}
-k^{I} y \\
0
\end{array}, \quad \hat{\Lambda}^{m}(x, y)=-k^{m} y,\right.
$$

where $k^{I}$ and $k^{m}$ are constants and we have included a minus sign for later convenience. $k^{I}, k^{m}$ will be further restricted below to ensure that (2.14) is in fact a large gauge transformation (preserving the boundary conditions of all fields). Using the split $\mathcal{I}=(I, \boldsymbol{\alpha})$ as in $(2.1)$ we have set $\hat{\Lambda}^{\boldsymbol{\alpha}}(x, y)=0$ to ensure that the Coulomb branch values $\left\langle\zeta^{\boldsymbol{\alpha}}\right\rangle=0$ in (2.5) are unchanged. This guarantees that we stay on the considered Coulomb branch; all the following discussions are performed on this background. The reduction ansätze (2.3) and (2.11) are also compatible with a gauge transformation (2.14) if one introduces the new quantities

$$
\tilde{r}=r, \quad \tilde{\zeta}^{I}=\zeta^{I}+\frac{k^{I}}{r}, \quad \tilde{\zeta}^{m}=\zeta^{m}+\frac{k^{m}}{r},
$$


and

$$
\left(\begin{array}{c}
\tilde{A}^{0} \\
\tilde{A}^{I} \\
\tilde{A}^{m} \\
\tilde{A}^{\alpha}
\end{array}\right)=\left(\begin{array}{cccc}
1 & 0 & 0 & 0 \\
-k^{I} & \delta_{J}^{I} & 0 & 0 \\
-k^{m} & 0 & \delta_{n}^{m} & 0 \\
\frac{1}{2} k^{K} k^{L} \mathcal{C}_{K L} b^{\alpha}+\frac{1}{2} k^{p} k^{q} b_{p q}^{\alpha} & -k^{K} \mathcal{C}_{K J} b^{\alpha} & -k^{p} b_{p n}^{\alpha} & \delta_{\beta}^{\alpha}
\end{array}\right) \cdot\left(\begin{array}{c}
A^{0} \\
A^{J} \\
A^{n} \\
A^{\beta}
\end{array}\right) .
$$

With (2.14) being compatible with (2.3) and (2.11) we mean that the form of the reduction ansatz after a gauge transformation is unchanged when using the tilded quantities.

Some additional remarks are in order here. First, it is important to stress that the simple shifts in the vector fields $\tilde{A}^{I}$ only occur for the Cartan direction. In the non-Cartan directions, i.e. for the vectors that are massive in the Coulomb branch, the non-Abelian structure of $G$ modifies the transformation rule. Second, while $\left\langle\zeta^{\boldsymbol{\alpha}}\right\rangle=0$ is preserved by (2.14) the actual values for $\left\langle\zeta^{I}\right\rangle$ do change in the vacuum. One therefore relates theories at different points on the Coulomb branch. In fact, this is a defining property of a large gauge transformation: they relate theories at different points in the vacuum manifold of the theory with the same properties, see e.g. [41]. Third, later on we will consider sixdimensional theories with $(1,0)$ supersymmetry arising in F-theory. These theories have $T_{\text {sd }}=1$ and $T_{\text {asd }} \equiv T$. Each six-dimensional anti-self-dual tensor is accompanied by a real scalar field in the multiplet. After dimensional reduction these scalar fields combine with $T$ of the $A^{\alpha}$ into vector multiplets. Importantly, it was found in $[9,36]$ that the redefinition of the five-dimensional scalar fields is precisely of the form compatible with (2.16) (see e.g. (3.30) in [9]). In other words, a gauge transformation (2.14) shifts both the vectors and scalars in a compatible fashion. We note that a similar story applies to circle compactifications from four to three space-time dimensions. In fact, the transformations (2.15) and (2.16) are equally valid for this latter case. As noted above the vectors $A^{\alpha}$ are the duals of the four-dimensional scalars $\hat{\rho}_{\alpha}$ appearing in (2.13).

Clearly, a gauge transformation (2.14) also requires to transform the Kaluza-Klein modes of all higher-dimensional charged fields. Given a matter state $\psi_{(n)}$ at Kaluza-Klein level $n$ in the representation $R$ of $G$ and with charge $q_{m}$ under $\hat{A}^{m}$ we first proceed as described after (2.7) and introduce eigenstates $\psi_{(n)}(w, q)$, where $w$ are the weights of $R$. The transformation (2.16) mixes these states as

$$
\psi_{(n)}(w, q) \mapsto \psi_{(\tilde{n})}(\tilde{w}, \tilde{q}), \quad\left(\begin{array}{c}
\tilde{n} \\
\tilde{w}_{I} \\
\tilde{q}_{m}
\end{array}\right)=\left(\begin{array}{ccc}
1 & -k^{J} & -k^{n} \\
0 & \delta_{I}^{J} & 0 \\
0 & 0 & \delta_{m}^{n}
\end{array}\right) \cdot\left(\begin{array}{c}
n \\
w_{J} \\
q_{n}
\end{array}\right) .
$$

Note that in general this transformation shifts the whole Kaluza-Klein tower, but there is still no state charged under $\tilde{A}^{\alpha}$. Furthermore, imposing that (2.17) is in fact a consistent reshuffling of the Kaluza-Klein states, which is necessary for invariance of the theory, imposes conditions on the constants $k^{I}$ and $k^{m}$ that are dependent on the spectrum of the theory. We will discuss the various choices and conditions in the following. 
Integer large gauge transformations. In (2.14) we have introduced gauge transformations that depend on the circle coordinate $y \sim y+2 \pi$. As mentioned before, these correspond to large gauge transformations around the circle if they preserve the circle boundary conditions of all fields and wind at least once around the circle. Let us now define what we mean by integer large gauge transformations. First, if we consider pure gauge theory without charged matter, we call a large gauge transformation to be integer if all $k^{I}$ and all $k^{m}$ are integers. Indeed, the degrees of freedom in (2.14) are in general characterized by elements in the homotopy groups

$$
\pi_{1}\left(\mathrm{U}(1)^{\mathrm{rk} G}\right) \cong \mathbb{Z}^{\mathrm{rk} G}, \quad \pi_{1}\left(\mathrm{U}(1)^{n_{\mathrm{U}(1)}}\right) \cong \mathbb{Z}^{n_{\mathrm{U}(1)}} .
$$

Clearly, (2.14) define maps from $S^{1}$ into the gauge group (which is purely Abelian on the Coulomb branch). These are precisely classified by the first homotopy group of the gauge group, which in the case at hand consists of tuples of integers.

If one now includes a charged matter spectrum the invariance of the boundary conditions of all these fields dictates the set of large gauge transformations. In these cases, the space of allowed $k^{I}$ and $k^{m}$ has to be quantized. In general, the $k^{I}$ and $k^{m}$ could still be integer or fractional depending on the weights and charges of the matter fields. However, for the transformations (2.14) to be an actual symmetry, i.e. a large gauge transformation, the following condition for each state $\hat{\psi}(R, q)$ has to be satisfied:

$$
k^{I} w_{I}+k^{m} q_{m} \in \mathbb{Z},
$$

where $w_{I}$ are the weights of $R$ and $q_{m}$ are the $\mathrm{U}(1)$ charges. This condition also arises from the transformation of the Kaluza-Klein level in (2.17) and ensures that $\tilde{n}$ is an integer, which implies equivalence of the full Kaluza-Klein towers of the compactified theory by a simple reshuffling. Now we are in the position to introduce our notion of integer large gauge transformations. They are spanned by pairs $\left(k^{I}, k^{m}\right)$ satisfying $(2.19)$ and one of the conditions

(I) $k^{m}=0$ and $k^{I} \in \mathbb{Z}$,

(II) $k^{m} \in \mathbb{Z}^{*}$ and $k^{I} w_{I} \in \mathbb{Q}$.

It is useful to comment on the class (II) of basis vectors. While all $w_{I}$ reside in an integer lattice and therefore do not violate (2.19) for integer $k^{I}$, the $\mathrm{U}(1)$ charges $q_{m}$ can be fractional. However, we will also consider the set of integer $k^{m}$ 's that allow a compensation of this fractional contribution to $(2.19)$ by an appropriate $k^{I}$-transformation which might be fractional.

Special fractional large gauge transformations. There is another set of large gauge transformations that will be of importance for us. If the arising representations in the spectrum of matter states is special, e.g. if the fundamental representation does not occur, also fractional $k^{I}$ might be allowed. More precisely, we also want to consider pairs $\left(k^{I}, k^{m}\right)$ satisfying (2.19) and

(III) $k^{m}=0$ and $k^{I}$ fractional. 
We call large gauge transformations satisfying (III) special fractional large gauge transformations. Note that the conceptual difference between (III) and (I), (II) is that there is always at least one integer quantity $k^{m}, k^{I}$ in (I) and (II).

It remains to consider the cases where also $k^{m}$ is fractional. As a concrete example this could be allowed if the spectrum has special charges such that $k^{m} q_{m}$ is integer for each state, although there are more general possibilities involving also the non-Abelian sector. We find, however, that models which allow for a fractional $k^{m}$ do not appear in our geometric considerations. For instance in the known F-theory examples there are always states that have minimal charge $0<q_{m} \leq 1$. Following some folk theorems (see e.g. [42, 43]) this might be true in any theory of quantum gravity. In this case the space of all large gauge transformations is spanned by $\left(k^{I}, k^{m}\right)$ satisfying (2.19) and either (I), (II) or (III).

Comments on one-loop Chern-Simons terms. The listed large gauge transformations are of key importance if one aims to study the anomalies of the six- or four-dimensional theory by using the circle compactified effective theory and its Chern-Simons terms. Let us combine all massless vectors in the Coulomb branch as $A^{\Sigma}=\left(A^{0}, A^{I}, A^{m}, A^{\alpha}\right)$. In five dimensions the gauge Chern-Simons terms then take the form

$$
S_{C S}^{(5)}=-\frac{1}{12} \int k_{\Sigma \Lambda \Gamma} A^{\Sigma} \wedge F^{\Lambda} \wedge F^{\Gamma}
$$

while in three dimensions they are given by

$$
S_{C S}^{(3)}=\int \Theta_{\Sigma \Lambda} A^{\Sigma} \wedge F^{\Lambda}
$$

The coefficients $k_{\Sigma \Lambda \Gamma}$ and $\Theta_{\Sigma \Lambda}$ are constants and can either arise directly in the dimensional reduction or be induced at one loop after integrating out all massive states. It was a key result of [18] that the one loop Chern-Simons terms precisely yield the higher-dimensional gauge anomaly conditions when transformed under (2.16) and (2.17) (see $[9,20]$ for earlier results on the four- and six-dimensional (mixed) gravitational anomalies). This can be traced back to the fact that the large gauge transformations are only a symmetry at oneloop if anomalies are cancelled. It appears that the Chern-Simons terms of the lowerdimensional effective theory capture all the required data to perform this test for the underlying higher-dimensional theory.

\section{On the systematics of F-theory compactifications}

In the previous sections we have discussed the compactification of gauge theories on a circle. Such theories prominently arise in the context of F-theory compactifications. In order to derive the effective action of F-theory on an elliptically-fibered Calabi-Yau geometry one is forced to use the duality to M-theory. More precisely, F-theory on a singular elliptically-fibered Calabi-Yau manifold, after an additional circle-reduction and moving to the Coulomb branch is dual to M-theory on the same but resolved manifold. Shrinking the elliptic fiber to zero size on the M-theory side corresponds to the decompactification limit of the circle on the F-theory side. Many properties of the F-theory effective action, like gauge 
symmetry, parts of the spectrum and Yukawa couplings, are encoded in the geometry of the elliptic fibration. A profound understanding of the latter therefore offers deep insights into the heart of F-theory compactifications. Noting that the M-theory to F-theory limit provides the correct approach to understanding the system, we have to suspect that the smooth Calabi-Yau geometry should share the symmetries of the gauge theories on a circle introduced in subsection 2.2. In the following we will therefore introduce some basics about the smooth F-theory geometries relevant to this work.

For the rest of this work we will study six-dimensional theories with $\mathcal{N}=(1,0)$ and four-dimensional theories with $\mathcal{N}=1$ supersymmetry. These arise as effective actions of $\mathrm{F}$ theory on elliptically fibered Calabi-Yau threefolds and fourfolds, respectively. Non-Abelian gauge symmetries are induced from singularities of the elliptic fiber at codimension-one loci in the base, while Abelian gauge factors are related to rational sections. The effective actions are accessed using the M-theory to F-theory limit. The circle reduced theories are thus compared with M-theory, or rather eleven-dimensional supergravity, compactified on the smooth Calabi-Yau manifold [1, 2, 9, 36, 40, 44-46]. In this manner the four-/sixdimensional supergravity data of the F-theory effective action is matched to geometric quantities using the three- or five-dimensional effective theories, respectively. It is important to realize that a classical matching procedure fails. In particular, for certain ChernSimons terms on the M-theory side one cannot find counterparts in the circle reduced supergravity at the classical level. The required corrections in the circle compactification arise at the one-loop level after integrating out the massive modes. As discussed briefly at the end of subsection 2.2 this process induces one-loop Chern-Simons terms and both reductions can be matched properly $[9,11,19,20,30,36]$. We highlight that, since oneloop induced Chern-Simons terms carry information about the number of matter fields, the matching to M-theory allows to translate information about the spectrum into the geometric data of the resolved space. This geometric perspective will be introduced next.

First let us establish some geometric notions of the Calabi-Yau manifold. We denote the resolved Calabi-Yau space by $\hat{Y}$. We assume that it constitutes an elliptic fibration over some base space $B$, with the corresponding projection given by $\pi: \hat{Y} \rightarrow B$. For this subsection we will assume that the fibration has at least one section. A set of linearly independent (minimal) rational sections of the elliptic fibration is denoted by $s_{0}, s_{m}, t_{r}$ where one arbitrary section $s_{0}$ is singled out as the so-called zero-section. The sections $t_{r}$ will be purely torsional, while the $s_{m}$ are assumed to be non-torsional. We will have to say more about rational sections and this distinction in section 4 . Furthermore, there might exist a divisor $S^{\mathrm{b}}$ in the base $B$ of the resolved space $\hat{Y}$ over which the fiber becomes reducible with the individual irreducible components intersecting as the (affine) Dynkin diagram of the gauge algebra. Fibering these over the corresponding codimension-one locus $S^{\mathrm{b}}$ in $B$ yields the blow-up divisors of $\hat{Y}$, which we denote by $D_{I}$.

In the following we define a basis of divisors $D_{\Lambda}=\left(D_{0}, D_{I}, D_{m}, D_{\alpha}\right)$ on the resolved space $\hat{Y}$ in the correct frame such that the corresponding gauge fields obtained from the expansion of the M-theory three-form

$$
C_{3}=A^{0} \wedge\left[D_{0}\right]+A^{m} \wedge\left[D_{m}\right]+A^{I} \wedge\left[D_{I}\right]+A^{\alpha} \wedge\left[D_{\alpha}\right]
$$


can be matched properly to the circle reduced theory. In this expression $[D]$ denotes the Poincaré-dual two-form to the divisor $D$ in $\hat{Y}$.

- Divisors $D_{\alpha}^{\mathrm{b}}$ of the base $B$ define the vertical divisors $D_{\alpha}:=\pi^{-1}\left(D_{\alpha}^{\mathrm{b}}\right)$ via pullback. For each $D_{\alpha}^{\mathrm{b}}$ in $B$ there is an axion in the four-dimensional F-theory compactification and an (anti-)self-dual tensor in the six-dimensional setting, respectively. Supersymmetry implies $T_{\mathrm{sd}}=1$ and thus we find

$$
\begin{aligned}
T_{\mathrm{asd}} & \equiv T=h^{1,1}(B)-1 \text { in six dimensions } \\
n_{\mathrm{ax}} & =h^{1,1}(B) \text { in four dimensions }
\end{aligned}
$$

with $T_{\mathrm{sd}}, T_{\mathrm{asd}}, n_{\mathrm{ax}}$ as defined in subsection 2.1.

For Calabi-Yau fourfolds it is also necessary to introduce vertical four-cycles $\mathcal{C}^{\alpha}:=$ $\pi^{-1}\left(\mathcal{C}_{\mathrm{b}}^{\alpha}\right)$, which are the pullbacks of curves $\mathcal{C}_{\mathrm{b}}^{\alpha}$ in the base intersecting the $D_{\alpha}^{\mathrm{b}}$ as

$$
\eta_{\alpha}{ }^{\beta}=D_{\alpha}^{\mathrm{b}} \cdot \mathcal{C}_{\mathrm{b}}^{\beta}
$$

with $\eta_{\alpha}{ }^{\beta}$ a full-rank matrix. For Calabi-Yau threefolds the analogous intersection matrix

$$
\eta_{\alpha \beta}:=D_{\alpha}^{\mathrm{b}} \cdot D_{\beta}^{\mathrm{b}}
$$

is used to raise and lower indices $\alpha, \beta$. The matrices (3.3) and (3.4) appear in the four- and six-dimensional Green-Schwarz terms (2.13) and (2.12), respectively.

For later convenience we also define the projection of two arbitrary divisors $D, D^{\prime}$ as

$$
\pi\left(D \cdot D^{\prime}\right):= \begin{cases}\left(D \cdot D^{\prime} \cdot \mathcal{C}^{\beta}\right) \eta_{\beta}^{-1}{ }^{\alpha} D_{\alpha} & \text { in three dimensions } \\ \left(D \cdot D^{\prime} \cdot D^{\alpha}\right) D_{\alpha} & \text { in five dimensions. }\end{cases}
$$

Furthermore we denote by $\pi_{\mathcal{M} I}$ the intersection number of a section $s_{\mathcal{M}}$ with a blowup divisor $D_{I}$ in the elliptic fiber $\mathcal{E}$ :

$$
\pi_{\mathcal{M} I}:=\left.\cap\left(s_{\mathcal{M}}, D_{I}\right)\right|_{\mathcal{E}}
$$

- We denote the divisor associated to the zero-section $s_{0}$ by $S_{0} \equiv \operatorname{Div}\left(s_{0}\right)$. The divisor $D_{0}$ is then defined by shifting $S_{0}$ as

$$
D_{0}=S_{0}-\frac{1}{2} \pi\left(S_{0} \cdot S_{0}\right)
$$

The corresponding vector $A^{0}$ in (3.1) is identified with the Kaluza-Klein vector in the circle reduced F-theory setting, i.e. with $A^{0}$ in (2.2).

- The $D_{I}$ denote the blow-up divisors and yield the Cartan gauge fields $A^{I}$ in (3.1). This implies that $I=1, \ldots, \operatorname{rank} G$. 
- Given a set of rational sections $s_{m}$ the U(1) divisors $D_{m}$ are defined via the Shioda map. Denote by $S_{m} \equiv \operatorname{Div}\left(s_{m}\right)$ the divisor associated to $s_{m}$. The Shioda map $D(\cdot)$ reads

$$
D\left(s_{m}\right) \equiv D_{m}=S_{m}-S_{0}-\pi\left(\left(S_{m}-S_{0}\right) \cdot S_{0}\right)+\pi_{m I} \mathcal{C}^{-1 I J} D_{J},
$$

where $\mathcal{C}_{I J}$ is the coroot intersection matrix (A.3) of $G$. The $D_{m}$ yield in (3.1) the Abelian gauge symmetries in the F-theory setting such that $m=1, \ldots, n_{\mathrm{U}(1)}$.

- The crucial property of the purely torsional sections $t_{r}$ is that they have no non-trivial image under the Shioda map. Denoting the divisors associated to $t_{r}$ by $T_{r}=\operatorname{Div}\left(t_{r}\right)$ one has $[47]$

$$
D\left(t_{r}\right)=T_{r}-S_{0}-\pi\left(\left(T_{r}-S_{0}\right) \cdot S_{0}\right)+\pi_{r I} \mathcal{C}^{-1 I J} D_{J}=0,
$$

which, as the other expressions above, should be read in homology.

By the Shioda-Tate-Wazir theorem $\left(D_{0}, D_{I}, D_{m}, D_{\alpha}\right)$ indeed form a basis of the NerónSeveri group of divisors ${ }^{3}$ (times $\mathbb{Q}$ ). Note also that the definition of the base divisor ensures

$$
\pi\left(D_{0} \cdot D_{0}\right)=0
$$

and the Shioda map enjoys the orthogonality properties

$$
\pi\left(D_{m} \cdot D_{\alpha}\right)=\pi\left(D_{m} \cdot D_{I}\right)=\pi\left(D_{m} \cdot D_{0}\right)=0,
$$

which are essential in order to perform the F-theory limit correctly. The blow-up divisors further satisfy the properties

$$
\pi\left(D_{I} \cdot D_{\alpha}\right)=\pi\left(D_{I} \cdot D_{0}\right)=0 .
$$

Via the matching of the M-theory compactification to the circle reduced theory the intersections of the divisor basis $\pi\left(D_{\Lambda} \cdot D_{\Sigma}\right) \equiv \pi\left(D_{\Lambda} \cdot D_{\Sigma}\right)^{\alpha} D_{\alpha}$ can be nicely related to four- and six-dimensional supergravity data

$$
\begin{aligned}
-\pi\left(D_{I} \cdot D_{J}\right)^{\alpha} & =\mathcal{C}_{I J} b^{\alpha}, \\
-\pi\left(D_{m} \cdot D_{n}\right)^{\alpha} & =b_{m n}^{\alpha},
\end{aligned}
$$

where $b^{\alpha}, b_{m n}^{\alpha}$ are the Green-Schwarz couplings appearing in (2.13) and (2.12). These relations hold both for Calabi-Yau three- and fourfolds. The Green-Schwarz coefficients $b^{\alpha}$ are equivalently obtained as

$$
S^{\mathrm{b}}=b^{\alpha} D_{\alpha}^{\mathrm{b}},
$$

where $S^{\mathrm{b}}$ was the divisor in $B$ supporting the non-Abelian gauge group. The remaining intersections are not directly relevant in the following discussions and may be found, for example, in $[9,11,18]$.

\footnotetext{
${ }^{3}$ For Calabi-Yau manifolds the Nerón-Severi group coincides with the Picard group, which is why we will identify both groups throughout this paper.
} 


\section{Arithmetic structure on fibrations with rational sections}

In this section we argue that the arithmetic structures of elliptic fibrations with multiple rational sections correspond to certain large gauge transformations introduced in subsection 2.2. The considered arithmetic is encoded by the so-called Mordell-Weil group of rational sections, which we introduce in more detail in subsection 4.1. In subsection 4.1 we also discuss how the geometric Mordell-Weil group law translates to a general group law for rational sections in terms of homological cycles. The free generators of Mordell-Weil group correspond to Abelian gauge symmetries in the effective F-theory action. In subsection 4.2 we show that group actions of the free part of the Mordell-Weil group are in one-to-one correspondence to specific integer large gauge transformations along the F-theory circle. A similar analysis for the torsion subgroup is performed in subsection 4.3. We find that it precisely captures special fractional non-Abelian gauge transformations introduced in subsection 2.2, which indicates the presence of a non-simply connected non-Abelian gauge group.

\subsection{On the Mordell-Weil group and its divisor group law}

A famous arithmetic structure on an elliptic curve is encoded by the Mordell-Weil group. The Mordell-Weil group is formed by the rational points of an elliptic curve endowed with a certain geometric group law (see, e.g. [48]). The rational points on the generic elliptic fiber of an elliptic fibration $Y$ directly extend to rational sections and form a finitely generated Abelian group, which is called the Mordell-Weil group of rational sections $\mathrm{MW}(Y)$. Thus it splits into a free part and a torsion subgroup

$$
\operatorname{MW}(Y) \cong \mathbb{Z}^{\operatorname{rank} \operatorname{MW}(Y)} \oplus \mathbb{Z}_{k_{1}} \oplus \ldots \oplus \mathbb{Z}_{k_{n_{\text {tor }}}}
$$

Having chosen one (arbitrary) zero section as the neutral element of the Mordell-Weil group, rank $\mathrm{MW}(Y)$ rational sections generate the free part and $n_{\text {tor }}$ rational sections generate the torsion subgroup. The precise group law on the generic fiber (in Weierstrass form) may be looked up for example in [48]. We denote the addition of sections $s_{1}, s_{2}$ using the Mordell-Weil group law by ' $\oplus$ ', i.e. we write $s_{3}=s_{1} \oplus s_{2}$ with $s_{3}$ being the new rational section. Since, as noted before, the rational sections $s_{\mathcal{M}}$ of an elliptic fibration define divisors $S_{\mathcal{M}} \equiv \operatorname{Div}\left(s_{\mathcal{M}}\right)$, we will investigate how the group law is translated to divisors. More precisely, we will derive the divisor class

$$
\operatorname{Div}\left(s_{1} \oplus k s_{2}\right), \quad k \in \mathbb{Z},
$$

where $k s_{2}=s_{2} \oplus \ldots \oplus s_{2}$ with $k$ summands. In contrast the addition in homology of divisor classes associated to sections is denoted by ' + ' . Extending the treatment in [5] the group law, written in homology, is uniquely determined by the three conditions:

1. The Shioda map $D\left(s_{\mathcal{M}}\right)$ introduced in $(3.8)$ is a homomorphism from the MordellWeil group to the Nerón-Severi group (times $\mathbb{Q}$ )

$$
D\left(s_{1} \oplus k s_{2}\right)=D\left(s_{1}\right)+k D\left(s_{2}\right) .
$$


2. A section $s_{\mathcal{M}}$ intersects the generic fiber $\mathcal{E}$ exactly once

$$
S_{\mathcal{M}} \cdot \mathcal{E}=1
$$

3. In the base $B$ a divisor $S_{\mathcal{M}}$ associated to a section squares to the canonical class of the base $K$, i.e.

$$
\pi\left(S_{\mathcal{M}} \cdot S_{\mathcal{M}}\right)=K
$$

The group law for two sections $s_{1}, s_{2}$ on the level of divisors then takes the form

$$
\operatorname{Div}\left(s_{1} \oplus k s_{2}\right)=S_{1}+k\left(S_{2}-S_{0}\right)-k \pi\left(\left(S_{1}-k S_{0}\right) \cdot\left(S_{2}-S_{0}\right)\right),
$$

where $S_{0}$ denotes the divisor associated to the zero-section $s_{0}$. We stress that we assumed that blow-up divisors do not contribute to the group-law. This can be derived easily in a general ansatz by enforcing that $\operatorname{Div}\left(s_{1} \oplus s_{0}\right)=\operatorname{Div}\left(s_{0} \oplus s_{1}\right)=\operatorname{Div}\left(s_{1}\right)$. In other words, the appearance of blow-up divisors in the ansatz always violates the Abelian structure of the group.

It is well known that the Shioda map as an injective homomorphism (3.8) transfers this group structure to the Nerón-Severi group (times $\mathbb{Q}$ ) of divisors modulo algebraic equivalence. Therefore it is reasonable to ask how a Mordell-Weil group action on the elliptic fibration effects the circle-reduced supergravity. We will find that the free part of the Mordell-Weil group corresponds to certain Abelian large gauge transformations, while the torsion subgroup manifests in special fractional non-Abelian large gauge transformations. As briefly mentioned at the end of subsection 2.2 and discussed in more detail in [18] these arithmetic structures allow to establish the cancellation of all pure Abelian and mixed Abelian-non-Abelian gauge anomalies in the effective field theory of F-theory.

\subsection{The free part of the Mordell-Weil group}

Let us first consider the free part of the Mordell-Weil group. On the elements of the MordellWeil basis, consisting of the zero-section $s_{0}$, the free generators $s_{m}$, and the torsional generators $t_{r}$, we now perform a number of $k^{m} \in \mathbb{Z}$ shifts into the directions of the free generators $s_{m}$, i.e. we find a new Mordell-Weil basis given by

$$
\tilde{s}_{0}:=s_{0} \oplus k^{n} s_{n}, \quad \tilde{s}_{m}:=s_{m} \oplus k^{n} s_{n}, \quad \tilde{t}_{r}:=t_{r} \oplus k^{n} s_{n},
$$

where $k^{n} s_{n}=k^{1} s_{1} \oplus \ldots \oplus k^{n_{\mathrm{U}(1)}} s_{n_{\mathrm{U}(1)}}$ and each summand $k^{1} s_{1}, k^{2} s_{2}, \ldots$ is evaluated using the Mordell-Weil group law. Our goal will be to translate these shifts to the divisor basis $D_{\Lambda}=\left(D_{0}, D_{I}, D_{m}, D_{\alpha}\right)$ introduced in section 3 , and then identify the corresponding large gauge transformation.

We use the formula (4.6) to identify the change in the definition of the $\mathrm{U}(1)$ divisors $D_{m}$, the Cartan divisors $D_{I}$ and the base divisor $D_{0}$. Note that the divisors $\tilde{D}_{\alpha}=D_{\alpha}$ are 
unchanged under this transformation. Explicitly we find

$$
\begin{aligned}
& \tilde{D}_{m}=D_{m}-k^{n} \pi\left(D_{n} \cdot D_{m}\right), \\
& \tilde{D}_{I}=D_{I}-k^{K} \pi\left(D_{K} \cdot D_{I}\right), \\
& \tilde{D}_{0}=D_{0}+k^{n} D_{n}+k^{J} D_{J}-\frac{k^{n} k^{p}}{2} \pi\left(D_{n} \cdot D_{p}\right)-\frac{k^{J} k^{L}}{2} \pi\left(D_{J} \cdot D_{L}\right) .
\end{aligned}
$$

where we have defined

$$
k^{I}:=-k^{n} \pi_{n J} \mathcal{C}^{-1 J I} .
$$

Using the expressions (3.13) one further evaluates

$$
\left(\begin{array}{c}
\tilde{D}_{0} \\
\tilde{D}_{I} \\
\tilde{D}_{m} \\
\tilde{D}_{\alpha}
\end{array}\right)=\left(\begin{array}{cccc}
1 & k^{J} & k^{n} & \frac{k^{q} k^{p}}{2} b_{q p}^{\beta}+\frac{k^{J} k^{L}}{2} \mathcal{C}_{J L} b^{\beta} \\
0 & \delta_{I}^{J} & 0 & k^{K} \mathcal{C}_{I K} b^{\beta} \\
0 & 0 & \delta_{m}^{n} & k^{k} b_{m k}^{\beta} \\
0 & 0 & 0 & \delta_{\alpha}^{\beta}
\end{array}\right) \cdot\left(\begin{array}{c}
D_{0} \\
D_{J} \\
D_{n} \\
D_{\beta}
\end{array}\right) .
$$

It is now straightforward to check that inserting (4.10) and the large gauge transformations (2.16) with $\left(k^{m}, k^{I}\right)$ as in (4.7) and (4.9) one finds

$$
C_{3}=A^{\Lambda} \wedge\left[D_{\Lambda}\right]=\tilde{A}^{\Lambda} \wedge\left[\tilde{D}_{\Lambda}\right] .
$$

This implies that the Mordell-Weil shift (4.7) indeed induces a large gauge transformation discussed in subsection 2.2. More precisely, the pair $\left(k^{m}, k^{I}\right)$ are in general the basis vectors of type (II) and realize an Abelian large gauge transformation combined with a fractional non-Abelian large gauge transformation. It is also straightforward to check that the quantization condition (2.19) is satisfied for the pair $\left(k^{m}, k^{I}\right)$ inferred from the geometry. In fact, one finds

$$
k^{I} w_{I}+k^{n} q_{n}=k^{n}\left(-\pi_{n J} \mathcal{C}^{-1 J I} w_{I}+q_{n}\right)=k^{n}\left(S_{n}-S_{0}-\pi\left(\left(S_{n}-S_{0}\right) \cdot S_{0}\right)\right) \cdot \mathcal{C},
$$

where we have used that $w_{I}=D_{I} \cdot \mathcal{C}$ and $q_{m}=D_{m} \cdot \mathcal{C}$ are the charges of a matter state $\hat{\psi}\left(w_{I}, q_{m}\right)$ arising from an M2-brane wrapped on the curve $\mathcal{C}$. The condition (2.19) then follows from the fact that $k^{m} \in \mathbb{Z}$ and the appearing intersections between divisors $S_{n}, S_{0}$ and the curve $\mathcal{C}$ are always integral. From its definition (4.9) it is also clear that $k^{I}$ can be fractional due to the appearance of the inverse $\mathcal{C}^{-1 I J}$.

Let us make a few comments concerning the derivation and interpretation of (4.8) and (4.10). First, it seems from counting the number of conditions (4.7) and (4.8) that the former conditions cannot suffice to fix the complete transformation law. In fact, the shift of the non-Abelian Cartan divisors $D_{I}$ to $\tilde{D}_{I}$ is not immediately inferred from (4.7), but appears to be crucial to make the transformation well-defined. To derive (4.8) one first starts with the transformation to $\tilde{D}_{0}$ by evaluating $\tilde{S}_{0}=\operatorname{Div}\left(s_{0} \oplus k^{n} s_{n}\right)$ and using (3.7), which is straightforward. If one tries to proceed in a similar fashion for $\tilde{D}_{m}$ one realizes 
that the evaluation of the Shioda map (3.8) for $\tilde{D}_{m}$ in the transformed divisors formally requires also to use new $\tilde{D}_{I}$, which is not fixed by (4.7). However, note that the shift $(4.8 \mathrm{~b})$ is uniquely fixed by requiring that the $\tilde{D}_{I}$ again behave as genuine blow-up divisors. More precisely, we find that $(4.8 \mathrm{~b})$ is fixed if the three conditions

$$
\pi\left(\tilde{D}_{I} \cdot \tilde{D}_{\alpha}\right) \stackrel{!}{=} 0, \quad \pi\left(\tilde{D}_{I} \cdot \tilde{D}_{J}\right) \stackrel{!}{=} \pi\left(D_{I} \cdot D_{J}\right), \quad \pi\left(\tilde{D}_{I} \cdot \tilde{D}_{0}\right) \stackrel{!}{=} 0 .
$$

are to be satisfied for the new divisors. These are simply the conditions (3.12) and (3.13) in the $\tilde{D}_{\Lambda}$ basis. Having fixed $\tilde{D}_{I}$ the transformed $\tilde{D}_{m}$ in (4.8a) are determined uniquely.

Let us stress again that the non-Abelian part of this gauge transformation is absolutely essential. On the one hand it is non-zero if and only if $\pi_{m I} \neq 0$ for some $D_{I}$. On the other hand we find fractional U(1)-charges if and only if $\pi_{m I} \neq 0$. This can easily be seen in the Shioda map (3.8). The U(1) charge $q_{m}$ of an M2-brane state wrapping a holomorphic curve $\mathcal{C}$ is given by the intersection of $\mathcal{C}$ with $D_{m}$. A fractional contribution to the charge therefore can only arise from the last term in (3.8) since $\mathcal{C}^{-1 I J}$ in general has fractional components. In fact, since a section can only intersect nodes with Coxeter label equal to one, the last term in (3.8) always vanishes for the simple Lie algebras $E_{8}, F_{4}, G_{2}$, which do not have nodes with Coxeter label one, and they are precisely the only simple Lie algebras having integer $\mathcal{C}^{-1 I J} .4$ The Coxeter labels for the simple Lie algebras can be found in table 1. To put it in a nutshell, if and only if there are fractional $U(1)$ charges, the free Mordell-Weil group action induces Abelian large gauge transformations supplemented by non-zero fractional non-Abelian large gauge transformations. Effectively, in the presence of fractional U(1) charges, a pure Abelian large gauge transformation with integer winding $k^{m} \in \mathbb{Z}$ is in general ill-defined. What makes it well-behaved is precisely the additional contribution from the fractional non-Abelian large gauge transformation (which is by itself also ill-defined) which compensates for the fractional part in the Abelian sector. This matches the gauge theory discussion of subsection 2.2 .

It should be stressed that not all redefinitions (4.8) have an immediate geometric interpretation. Away from the singular loci in $B$ that are resolved, the transformation $D_{m} \rightarrow \tilde{D}_{m}$ is induced by the action of the Mordell-Weil group, which has a known geometric origin as addition of points in the fiber (see e.g. [48]). We are not familiar of how the latter geometric group law is extended to the non-Abelian singularities or to their resolutions. This prevents us from identifying a geometric interpretation of $D_{I} \rightarrow \tilde{D}_{I}$. Nevertheless the considered arithmetic operations are well-defined on the level of divisors and consistently include also the blow-up divisors, for example, in the Shioda map. This suffices to infer information about the effective theory after compactification on this space. We will encounter similar transformations in the general discussion of non-Abelian large gauge transformations in section 6 .

\subsection{The torsion part of the Mordell-Weil group}

In a similar spirit we can show that a non-trivial torsion subgroup in (4.1) is connected to special fractional non-Abelian large gauge transformations, i.e. to the basis vectors $\left(k^{I}, k^{m}\right)$

\footnotetext{
${ }^{4}$ This is related to the fact that the center of the corresponding universal covering group is trivial. We will elaborate more on this fact in the part about the torsion subgroup of the Mordell-Weil group.
} 


\begin{tabular}{|c|c|c|c|c|}
\hline algebra & Dynkin diagram & Coxeter labels & fund. representation & $\lambda_{G}$ \\
\hline$A_{n}$ & 1 & $(1,1,1, \ldots, 1,1)$ & $(1,0,0, \ldots, 0,0)$ & 1 \\
\hline$B_{n}$ & & $(1,2,2, \ldots, 2,2)$ & $(1,0,0, \ldots, 0,0)$ & 2 \\
\hline$C_{n}$ & & $(2,2,2, \ldots, 2,1)$ & $(1,0,0, \ldots, 0,0)$ & 1 \\
\hline$D_{n}$ & & $(1,2,2, \ldots, 2,1,1)$ & $(1,0,0, \ldots, 0,0,0)$ & 2 \\
\hline$E_{6}$ & & $(1,2,2,3,2,1)$ & $(0,0,0,0,0,1)$ & 6 \\
\hline$E_{7}$ & & $(2,2,3,4,3,2,1)$ & $(0,0,0,0,0,0,1)$ & 12 \\
\hline$E_{8}$ & & $(2,3,4,6,5,4,3,2)$ & $(0,0,0,0,0,0,0,1)$ & 60 \\
\hline$F_{4}$ & 1 & $(2,3,4,2)$ & $(0,0,0,1)$ & 6 \\
\hline$G_{2}$ & 1 & $(3,2)$ & $(1,0)$ & 2 \\
\hline
\end{tabular}

Table 1. Conventions for the simple Lie algebras.

of type (III) introduced in subsection 2.2. For a torsional section $t_{r}, r=1, \ldots, n_{\text {tor }}$, we use the key fact that its image under the Shioda map vanishes, cf. (3.9). As discussed in section 3 this implies that they do not define divisors that appear in the Kaluza-Klein expansion (3.1) and thus do not give rise to massless gauge fields in the effective theory.

Despite the fact that torsional sections do not define massless gauge fields in the effective field theory, the Mordell-Weil group action along these sections nevertheless results in a non-trivial transformation in the circle-reduced theory. In order to show this we perform $\tilde{k}^{r} \in \mathbb{Z}$ shifts along the torsional generators $t_{r}$ using the Mordell-Weil group law (4.6). The derivation proceeds in a similar fashion as the one in subsection 4.2. In fact, keeping in mind that $D\left(t_{r}\right)=0$ one can use (4.8) to infer

$$
\begin{aligned}
& \tilde{D}_{m}=D_{m} \\
& \tilde{D}_{I}=D_{I}-k^{K} \pi\left(D_{K} \cdot D_{I}\right) \\
& \tilde{D}_{0}=D_{0}+k^{J} D_{J}-\frac{k^{J} k^{L}}{2} \pi\left(D_{J} \cdot D_{L}\right),
\end{aligned}
$$


where we have defined, similar to (4.9), that

$$
k^{I}:=-\tilde{k}^{r} \pi_{r J} \mathcal{C}^{-1 J I} .
$$

Just as in subsection 4.2, in general $k^{I}$ will be fractional due to the appearance of the inverse matrix $\mathcal{C}^{-1 J I}$. In other words the transformations induced by $\tilde{k}^{r}$ correspond to special fractional non-Abelian large gauge transformations parametrized by pairs $\left(k^{I}, k^{m}=0\right)$, introduced as case (III) in subsection 2.2.

The fact that torsion in the Mordell-Weil group allows for the presence of special fractional non-Abelian large gauge transformations is not unexpected. As discussed in [47, 49], torsion in the Mordell-Weil group indicates that the gauge group is not simply connected, and therefore certain representations of the Lie algebra do not appear on the level of the group, i.e. the weight lattice of the group is coarser. Because of this fact also certain fractional non-Abelian large gauge transformations are compatible with the circle boundary conditions. Indeed the torsional shifts exhaust all possible fractional large gauge transformations, which is evident from considering the center of the universal covering group as in section 3.3 of [47].

\section{Introducing an arithmetic structure on fibrations with multi-sections}

In this section we aim to generalize the discussion of section 4 to Calabi-Yau geometries that admit a genus-one fibration that does not have a rational section. These setups always come with multi-sections that no longer cut out rational points of the genus-one fiber but rather roots, which are exchanged over branch cuts in the base $B$. On such genus-one fibrations with only multi-sections there is no known arithmetic structure analog to the Mordell-Weil group. However, our understanding of the F-theory effective action associated to such geometries, which will have U(1) gauge group factors if there is more than one independent multi-section, and the possibility to perform Abelian large gauge transformations in these setups suggest that an arithmetic structure should equally exist on genus-one fibrations. We will collect evidence for the existence of this structure, which we name the extended Mordell-Weil group, and study its key properties.

Our considerations will be driven by two facts. First, we will make use of the fact that a genus-one fibration with multi-sections can often be related by a geometric transition to elliptic fibrations with multiple rational sections. Physically this corresponds to an unHiggsing of Abelian gauge fields [15, 27, 28, 30-33]. Following the divisors through this transition we are able to reverse-engineer, on the level of divisor classes, the group law on the genus-fibration from the Mordell-Weil group law in the unHiggsed geometry. We note that, at this point, we can only determine the extended Mordell-Weil group law up to vertical divisors, which will be the task of subsection 5.1. This definition, however, will allow us to uniquely define a generalized Shioda map in subsection 5.2. The latter defines divisors associated to massless Abelian gauge symmetries from the generators of the postulated extended Mordell-Weil group, i.e. from the multi-sections. Finally, in subsection 5.3 we show that translations in the extended Mordell-Weil group correspond to Abelian large gauge transformations. 


\subsection{A group action for fibrations with multi-sections}

We now present an extension of the results from the last subsection to F-theory compactified on genus-one fibrations without section. These geometries come with multi-sections, which mark points in the elliptic fiber that are exchanged over branch cuts in the base $B$. If they mark a set of $n$ points in the fiber, we call the multi-section an $n$-section. For a genus-one fibration one can always birationally move to the Jacobian fibration, which replaces each independent $n$-section by a rational section and therefore constitutes an elliptic fibration. Importantly the genus-one fibration and its Jacobian describe the same F-theory effective action in four or six dimensions [25, 26]. It is therefore clear that the presence of at least two homologically independent multi-sections indicates the existence of massless $\mathrm{U}(1)$ gauge fields in the four- or six-dimensional F-theory effective field theory. ${ }^{5}$ In particular, the associated Jacobian fibration of a genus-one fibration with more than one multi-section will have a non-trivial Mordell-Weil group. One can therefore ask how to identify the divisor classes associated to massless U(1) gauge symmetries already in the genus-one fibrations. This is relevant e.g. for the computation of U(1)-charges or the computation of anomaly coefficients. Furthermore, we will argue for the existence of a group law for multi-sections.

To address these issues we first have to introduce some additional facts about fibrations with multi-sections and state our assumptions. First, recall that an $n$-section $s^{(n)}$ with divisor class $S^{(n)}=\operatorname{Div}\left(s^{(n)}\right)$ fulfills

$$
S^{(n)} \cdot f=n
$$

where $f$ is the class of the genus-one fiber. This implies that

$$
S^{(n)} \cdot D_{\alpha} \cdot D_{\beta}=n D_{\alpha}^{b} \cdot D_{\beta}^{b}, \quad S^{(n)} \cdot D_{\alpha} \cdot D_{\beta} \cdot D_{\gamma}=n D_{\alpha}^{b} \cdot D_{\beta}^{b} \cdot D_{\gamma}^{b},
$$

where the first equation applies for threefolds and the second for fourfolds. Second, note that it is always possible to find a basis of multi-sections in homology that are all of the same degree [26], i.e. they cut out the same number of points in the fiber. We denote the number of such multi-sections by $n_{\mathrm{ms}}$ and assume $n_{\mathrm{ms}} \geq 2$. We denote such a basis of $n$-sections by $s_{0}^{(n)}, s_{m}^{(n)}, m=1, \ldots, n_{\mathrm{ms}}-1$ and demand that it is minimal in the sense that there does not exist any multi-section in the geometry that cuts out $n-1$ or fewer points. ${ }^{6}$ We have singled out an arbitrary multi-section which we labelled by 0 . The divisors associated to these sections are denoted by $S_{0}^{(n)}=\operatorname{Div}\left(s_{0}^{(n)}\right)$ and $S_{m}^{(n)}=\operatorname{Div}\left(s_{m}^{(n)}\right)$ in accord with our previous notation.

To propose a group law we will work with the following assumption for genus-one fibrations throughout this section:

- We assume that there exists a specialization of the complex structure of the fibration such that each $n$-section $s_{0}^{(n)}$ and $s_{m}^{(n)}$ splits into $n$ sections $s_{0}^{1}, \ldots, s_{0}^{n}$ and $s_{m}^{1}, \ldots, s_{m}^{n}$. After resolving the singularities in the new geometry we will denote the resulting space

\footnotetext{
${ }^{5} \mathrm{~A}$ single multi-section gives one massless $\mathrm{U}(1)$ in the three- or five-dimensional effective theory, which captures the degree of freedom of the circle Kaluza-Klein vector.

${ }^{6}$ From now on we will always require that the considered basis of multi-sections is of this type.
} 
by $\hat{Y}_{\mathrm{uH}}$, where we indicate that this geometry captures the unHiggsing from a fieldtheoretic point of view. In the following we impose that the rational sections $s_{0}^{1}, \ldots, s_{0}^{n}$ and $s_{m}^{1}, \ldots, s_{m}^{n}$ are the generators of the Mordell-Weil group supplemented by the zero section of the elliptic fibration $\hat{Y}_{\mathrm{uH}}$. We expect, however, that the following discussion can be extended to the more general situation in which these rational sections only generate a sub-lattice of the Mordell-Weil lattice. With this simplification, the divisor homology groups of $\hat{Y}$ and $\hat{Y}_{\mathrm{uH}}$ are generated as follows:

$$
H_{p}(\hat{Y})=\left\langle S_{0}^{(n)}, S_{m}^{(n)}, D_{\alpha}\right\rangle, \quad H_{p}\left(\hat{Y}_{\mathrm{uH}}\right)=\left\langle S_{0}^{1}, \ldots, S_{0}^{n}, S_{m}^{1}, \ldots, S_{m}^{n}, D_{\alpha}^{\prime}\right\rangle
$$

where $p=4$ for Calabi-Yau threefolds and $p=6$ for Calabi-Yau fourfolds. Note that we will in the following assume that the theory has no non-Abelian gauge groups. In other words, we do not include exceptional divisors in (5.3).

- We also introduce an unHiggsing map $\varphi$ from the divisors of $\hat{Y}$, i.e. the fibrations admitting multi-sections, to the divisors of $\hat{Y}_{\mathrm{uH}}$,

$$
\varphi: \quad H_{p}(\hat{Y}) \hookrightarrow H_{p}\left(\hat{Y}_{\mathrm{uH}}\right)
$$

Here we have indicated that the map is injective. In addition, we require it to be an injective ring homomorphism from the full intersection ring on $\hat{Y}$ into that of $\hat{Y}_{\mathrm{uH}}$. This map is defined to identify the $n$-sections with $n$ sections on the divisor level:

$$
\varphi\left(S_{0}^{(n)}\right)=S_{0}^{1}+\cdots+S_{0}^{n}, \quad \varphi\left(S_{m}^{(n)}\right)=S_{m}^{1}+\cdots+S_{m}^{n} .
$$

We do not consider torsional sections in the following discussion. We furthermore assume that the map $\varphi$ acts trivially on the remaining divisors $D_{\alpha}$ and is linear on the vector space of divisors, i.e.

$$
\varphi\left(\nu^{i} S_{i}^{(n)}+\nu^{\alpha} D_{\alpha}\right)=\nu^{i} S_{i}^{1}+\cdots+\nu^{i} S_{i}^{n}+\nu^{\alpha} D_{\alpha}^{\prime}
$$

for some constants $\left(\nu^{i}, \nu^{\alpha}\right)$. Note that $D_{\alpha}$ and $D_{\alpha}^{\prime}$ actually define the same divisor classes, since they both ascend form the same divisors in the base $B$ common to both $\hat{Y}$ and $\hat{Y}_{\mathrm{uH}}$.

Note that that only a single example of a geometry with more than one independent multi-section has been studied in the literature [15], which is given by an embedding of the fiber as a hypersurface into $\mathbb{P}^{1} \times \mathbb{P}^{1}$. In these setups one finds two independent twosections, which do indeed split into four sections in the prescribed way by blowing up the fiber ambient space to $d P_{3}{ }^{7}$

Let us make the following preliminary ansatz for a group structure placed on the set of multi-sections written down in homology similar to (4.6): choose one $n$-section $s_{0}^{(n)}$ as what

\footnotetext{
${ }^{7}$ It is important to notice that the two toric two-sections of $\mathbb{P}^{1} \times \mathbb{P}^{1}$ do not exclusively split into the four toric sections of $d P_{3}$. One rather has to pick four appropriate elements of the Mordell-Weil lattice of the blow-up that are not necessarily torically realized.
} 


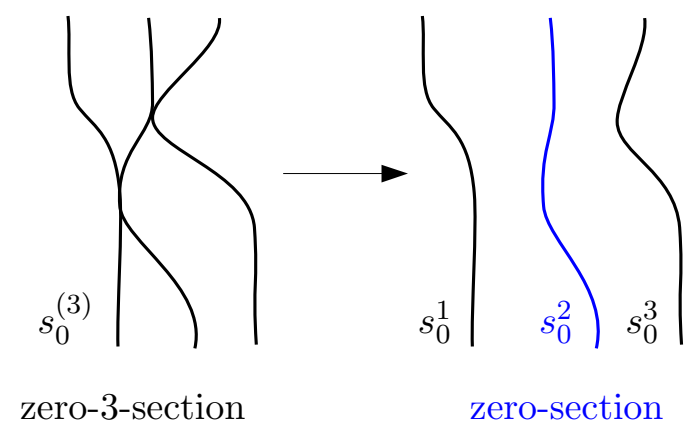

Figure 2. The zero- $n$-section is chosen to contain the zero-section after unHiggsing to a setting with rational sections only.

we call the zero- $n$-section or zero-multi-section. Then two arbitrary $n$-sections $s_{1}^{(n)}, s_{2}^{(n)}$ are added according to

$$
\operatorname{Div}\left(s_{1}^{(n)} \oplus k s_{2}^{(n)}\right):=S_{1}^{(n)}+k\left(S_{2}^{(n)}-S_{0}^{(n)}\right)+\lambda^{\alpha} D_{\alpha} .
$$

Making the definition (5.7) precise would require to determine the constants $\lambda^{\alpha}$. However, we will argue in the following that these are not uniquely determined, which can be traced back to the fact that there exist divisor classes corresponding to genuine multi-sections that differ only in their vertical parts induced by the base homology. This implies that we need to talk about equivalence classes [.] of divisor classes of multi-sections defined modulo vertical part. Furthermore, we will in the following provide evidence that $\operatorname{Div}\left(s_{1}^{(n)} \oplus k s_{2}^{(n)}\right)$ defines a divisors class representing an actual $n$-section in the geometry when neglecting the vertical part. Let us stress again that our approach just allows us to investigate how the group law for multi-section is defined in terms of homology classes.

We first want to provide evidence that there is indeed a multi-section associated to $\tilde{S}^{(n)} \equiv \operatorname{Div}\left(s_{1}^{(n)} \oplus k s_{2}^{(n)}\right)$ as defined in (5.7). In order to do that we will check in which ways $\varphi\left(\tilde{S}^{(n)}\right)$ can split into a sum of $n$ sections in the homology of $\hat{Y}_{\mathrm{uH}}$. Let us denote such a set of $n$ linear independent sections of $\hat{Y}_{\mathrm{uH}}$ by $\left\{\hat{s}^{i}\right\}$, and demand that

$$
\varphi\left(S_{1}^{(n)}+k\left(S_{2}^{(n)}-S_{0}^{(n)}\right)+\lambda^{\alpha} D_{\alpha}\right) \stackrel{!}{=} \sum_{i=1}^{n} \hat{S}^{i},
$$

where $\left\{\hat{S}^{i}=\operatorname{Div}\left(\hat{s}^{i}\right)\right\}$ is the associated set of linearly independent divisors in $\hat{Y}_{\mathrm{uH}}$. It turns out that there are infinitely many possibilities to define an appropriate set of sections $\left\{\hat{s}^{i}\right\}$. For example, choosing one arbitrary element $s_{0}^{l}$ (for fixed $l$ ) as the zero-section (see figure 2 where e.g. $l=2$ ), there is the very simple choice

$$
\hat{s}^{i}:=s_{1}^{i} \oplus k s_{2}^{i} \ominus k s_{0}^{i},
$$

which gives the right structure (5.8) upon using (5.6) and the conventional Mordell-Weil group law (4.6). Clearly, the ansatz (5.9) allows us to fix the $\lambda^{\alpha}$ specifying the vertical part in (5.7). The existence of an appropriate set of $\hat{s}^{i}$ indicates that there is indeed a multisection in the divisor class $\operatorname{Div}\left(s_{1}^{(n)} \oplus k s_{2}^{(n)}\right)$, when fixing the $\lambda^{\alpha}$ via (5.8), (5.9) and (4.6). 


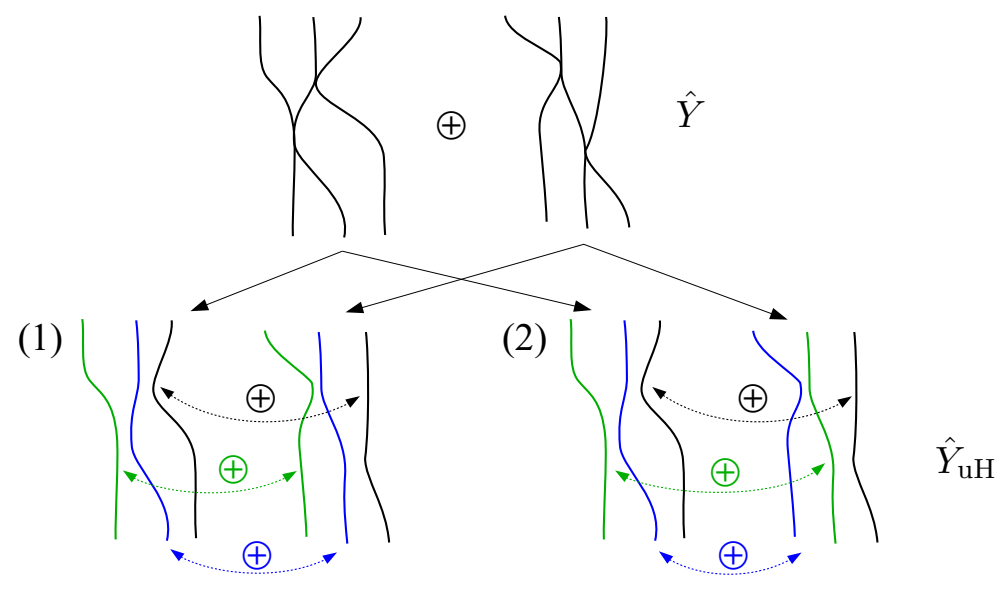

Figure 3. Moving from $\hat{Y}$ to the unHiggsed phase $\hat{Y}_{\mathrm{uH}}$ there are in general many ways to add the individual sections. We schematically indicate two choices in (1) and (2), which in general differ by their vertical parts when considering the associated multi-section.

However, using merely the existence of sections $\hat{s}^{i}$ in $\hat{Y}_{\mathrm{uH}}$ satisfying (5.8) does not seem to fix the class $\operatorname{Div}\left(s_{1}^{(n)} \oplus k s_{2}^{(n)}\right)$ uniquely. In fact, other appropriate sets $\left\{\hat{s}^{i}\right\}$ can be obtained if one picks two arbitrary sections out of $\left\{\hat{s}^{i}\right\}$ and adds a third arbitrary chosen section to one of the latter while subtracting it from the other one by using the MordellWeil group law on $\hat{Y}_{\mathrm{uH}}$. Such a freedom of choice is schematically depicted in figure 3 . The set $\left\{\hat{s}^{i}\right\}$ can be used to satisfy (5.8), but will generally yield a different set of constants $\lambda^{\alpha}$ compared to the choice (5.9). In other words, the contribution from vertical divisors in (5.7) is a priori not uniquely fixed by the compatibility conditions that we impose. This should be contrasted with the situation for genuine sections (i.e. $n=1$ ), in which this part is fixed by demanding that the section squares to the canonical class in the base of the elliptic fibration. For multi-sections this relation to the canonical class of the base is in general not valid. ${ }^{8}$ In fact, for concrete examples one can verify that there exist multisections that differ only by their vertical parts. ${ }^{9}$ Therefore, there is no immediate way that we are aware of to fix the vertical part of a multi-section. As stressed above this also reflects our inability to give a unique choice for the splitting (5.8) and the fixation of the constants $\lambda^{\alpha}$ in (5.7).

It is therefore natural to define the group law (5.7) only in terms of equivalence classes of multi-sections modulo vertical divisors as

$$
\widehat{\operatorname{Div}}\left(\left[s_{1}^{(n)}\right] \oplus k\left[s_{2}^{(n)}\right]\right):=\left[S_{1}^{(n)}+k\left(S_{2}^{(n)}-S_{0}^{(n)}\right)\right] .
$$

\footnotetext{
${ }^{8}$ This can also be understood after applying the map $\varphi$ to $\hat{Y}$, since two of the individual sections $s_{i}^{1}, s_{i}^{2}$ arising from the $i$ th $n$-section can have non-trivial intersection.

${ }^{9}$ One can consider for example fibrations where the fiber is embedded as a hypersurface into $\mathbb{P}^{1} \times \mathbb{P}^{1}$. In these setups one can find four toric multi-sections from which two are independent, and the other two do indeed differ by vertical parts from the latter in certain examples.
} 
In this expression we indicate that the divisors as well as the multi-sections should only be considered modulo vertical parts. $\widehat{D i v}$ maps between equivalence classes of multi-sections and equivalence classes of divisors and reduces on representatives to Div. Let us stress that this does not imply that one can add arbitrary vertical divisors to the right-hand side of this equation and find an actual multi-section in the geometry. The formulation in (5.10) loses some information about divisor classes supporting multi-sections. Crucially, this information turns out to be irrelevant in the discussion of the generalized Shioda map and therefore does not affect the considered physical implications of the setup.

\subsection{The generalized Shioda map}

In order to investigate the physical implications of the group law studied in subsection 5.1 for the effective field theory we first need to define a generalized Shioda map for multisections to be defined in this section. Recall that the considered multi-sections were arbitrarily split as $s_{0}^{(n)}, s_{m}^{(n)}, m=1, \ldots, n_{\mathrm{ms}}-1$, where we called $s_{0}^{(n)}$ the zero- $n$-section. The generalized Shioda map associates divisors $D_{m}^{(n)} \equiv D\left(s_{m}^{(n)}\right)$ to the $n$-sections $s_{m}^{(n)}$. In addition, one has to define a map from $s_{0}^{(n)}$ to a divisor $D_{0}^{(n)}$ generalizing (3.7), also to be defined below. The Poincaré-dual two-forms $\left[D_{0}^{(n)}\right]$ and $\left[D_{m}^{(n)}\right]$ can then appear in the Kaluza-Klein expansion of the M-theory three-form $C_{3}$ generalizing (3.1) as

$$
C_{3}=A^{\alpha} \wedge\left[D_{\alpha}\right]+\hat{A}^{0} \wedge\left[D_{0}^{(n)}\right]+\hat{A}^{m} \wedge\left[D_{m}^{(n)}\right]
$$

where we recall that we assume the absence of exceptional divisors $D_{I}$ associated to a non-Abelian gauge group in this section. The vectors $\hat{A}^{m}$ correspond to $\mathrm{U}(1)$ gauge fields in the six- or four-dimensional effective theory, while $\hat{A}^{0}$ will contain the degree of freedom arising from the Kaluza-Klein vector along the circle when performing the F-theory up-lift from five/three to six/four dimensions.

If we have $n_{\mathrm{ms}} \geq 2$, as we will assume in the following, we have to define generalized Shioda maps yielding the $\mathrm{U}(1)$ divisors $D_{m}^{(n)}$. To this end, we once again borrow results from the unHiggsed geometry $\hat{Y}_{\mathrm{uH}}$. As was explained in [15, 27, 28, 30-33] the transition from the unHiggsed geometry $\hat{Y}_{\mathrm{uH}}$ to the genus-one fibration is described by a Higgsing in the effective five- or three-dimensional field theory of certain matter states charged under a linear combination of the $n \times n_{\mathrm{ms}}$ Abelian gauge fields. We aim to find the proper linear combinations of these $\mathrm{U}(1) \mathrm{s}$ that constitute the massless $\mathrm{U}(1)$ vectors after the Higgsing. To begin with we consider the divisor classes $D_{m, l}^{\prime}$ on $\hat{Y}_{\mathrm{uH}}$ given by

$$
D_{m, l}^{\prime}:=\sum_{i=1}^{n} D_{m}^{i}-\sum_{\substack{i=1 \\ i \neq l}}^{n} D_{0}^{i},
$$

where $D_{m}^{i} \equiv D\left(s_{m}^{i}\right), D_{0}^{i}=D\left(s_{0}^{i}\right)$ denote the Shioda maps of $s_{m}^{i}, s_{0}^{i}$, with $i \neq l$, and we have chosen an element $s_{0}^{l}$ (fixed $l$ ) as the zero-section on $\hat{Y}_{\mathrm{uH}}$. Note that the ansatz (5.12) is invariant under the exchange of all components $s_{m}^{i}, s_{0}^{i}$ of a given multi-section $s_{m}^{(n)}$ and the zero-multi-section $s_{0}^{(n)}$, respectively, and it is consistent with the map $\varphi$ as defined in (5.5). In contrast, the definition (5.12) of $D_{m, l}^{\prime}$ still depends on the choice of the zero-section 
$s_{0}^{l}$ and is thus not invariant under the exchange of components of the zero-multi-section $s_{0}^{(n)}$. We therefore included an additional index $l$ in the notation. Inserting the explicit expressions for the Shioda maps $D_{m}^{i}, D_{0}^{i}$ and using (5.5) we obtain

$$
D_{m, l}^{\prime}=\varphi\left(S_{m}^{(n)}-S_{0}^{(n)}\right)-\pi\left(\varphi\left(S_{m}^{(n)}-S_{0}^{(n)}\right) \cdot S_{0}^{l}\right) .
$$

Thus it is clear that the expression (5.13) is still problematic if one wants to move solely to the phase of the genus-one fibration, since (5.13) manifestly depends on the choice of the zero-section $s_{0}^{l}$ on $\hat{Y}_{\mathrm{uH}}$. However, as it follows from the discussion of section 4 and was investigated already in [18], different choices of the zero-section are just related by large gauge transformations in the effective field theory. Therefore it seems logical to treat all phases with different zero-section $s_{0}^{l}$ on equal footing. We thus average over all these choices and use again (5.5) to obtain

$$
\frac{1}{n} \sum_{l} D_{m, l}^{\prime}=\varphi\left(S_{m}^{(n)}-S_{0}^{(n)}\right)-\frac{1}{n} \pi\left(\varphi\left(S_{m}^{(n)}-S_{0}^{(n)}\right) \cdot \varphi\left(S_{0}^{(n)}\right)\right) .
$$

Since $\varphi$ is a ring homomorphism and all pieces lie in the image of $\varphi$, we can now drop the map $\varphi$ in this expression and consistently define a generalized Shioda map $D_{m}^{(n)}$ for the multi-section $s_{m}^{(n)}$ without reference to an unHiggsed phase

$$
D_{m}^{(n)}:=S_{m}^{(n)}-S_{0}^{(n)}-\frac{1}{n} \pi\left(\left(S_{m}^{(n)}-S_{0}^{(n)}\right) \cdot S_{0}^{(n)}\right) .
$$

By construction it is evident that $\mathrm{U}(1)$ charges $q_{m}$ of matter in the genus-one fibration are calculated by intersecting the associated curves with $D_{m}^{(n)}$. Note that this intersection is independent of the vertical contribution in $D_{m}$. Furthermore (5.15) is a generalization of the map given in [15] (without the factor $\frac{1}{n}$ ), where the authors consider fibers embedded into $\mathbb{P}^{1} \times \mathbb{P}^{1}$. We expect that our definition of $D_{m}^{(n)}$ yields the correct $\mathrm{U}(1)$ divisors in order to study the effective field theory of F-theory on genus-one fibrations directly without explicit reference to an unHiggsed geometry $\hat{Y}_{\mathrm{uH}}$ or the Jacobian of $\hat{Y}$. Exploring this effective theory in detail is however beyond the scope of the present paper.

Further indication that $D_{m}^{(n)}$ is an important object of the genus-one fibration is provided by the fact that the definition (5.15) only depends on the equivalence classes $\left[S_{m}^{(n)}\right],\left[S_{0}^{(n)}\right]$. Indeed, it is easy to check that (5.15) even provides a homomorphism from the generalized Mordell-Weil group (5.10) to the Nerón-Severi group. Note that both of these conditions are extremely restrictive.

In a similar fashion we can construct the divisor $D_{0}^{(n)}$ appearing in (3.1). It is the cycle that is dual to the massless linear combination of the Kaluza-Klein vector and a set of $n-1$ $\mathrm{U}(1)$ vectors that are massive in the higher-dimensional theory [30]. These correspond to the individual constituents of the zero-multi-section under the splitting (5.5). In analogy to (5.12) we first make the ansatz

$$
D_{0, l}^{\prime}:=n D_{0}+\sum_{\substack{i=1 \\ i \neq l}}^{n} D_{0}^{i}
$$


where $D_{0}^{i}=D\left(s_{0}^{i}\right)$ are the Shioda maps with a chosen zero-section $s_{0}^{l}$. This expression is again invariant under the exchange of the individual sections $s_{0}^{i}$ modulo vertical divisors. We stress that $D_{0}$ denotes the divisor yielding the Kaluza-Klein vector in $\hat{Y}_{\mathrm{uH}}$ and is therefore given as in (3.7) by

$$
D_{0}=S_{0}^{l}-\frac{1}{2} \pi\left(S_{0}^{l} \cdot S_{0}^{l}\right)
$$

Using in (5.16) the explicit expressions for the Shioda maps as well as (5.17) and (5.5) we obtain

$$
D_{0, l}^{\prime}=\varphi\left(S_{0}^{(n)}\right)+\frac{n}{2} K-\pi\left(\varphi\left(S_{0}^{(n)}\right) \cdot S_{0}^{l}\right)
$$

Averaging over all zero-section choices as in (5.14) we get

$$
\frac{1}{n} \sum_{l} D_{0, l}^{\prime}=\varphi\left(S_{0}^{(n)}\right)+\frac{n}{2} K-\frac{1}{n} \pi\left(\varphi\left(S_{0}^{(n)}\right) \cdot \varphi\left(S_{0}^{(n)}\right)\right) .
$$

Now we can drop the map $\varphi$ using the similar arguments as above, we are able to define $D_{0}^{(n)}$ as

$$
D_{0}^{(n)}:=S_{0}^{(n)}+\frac{n}{2} K-\frac{1}{n} \pi\left(S_{0}^{(n)} \cdot S_{0}^{(n)}\right),
$$

which is a generalization of (3.7).

\subsection{Extended Mordell-Weil group and large gauge transformations}

In this final subsection we show that, similar to the genuine Mordell-Weil group of rational sections, translations in the extended Mordell-Weil group lattice are in one-to-one correspondence with Abelian large gauge transformations in the effective field theory. As before, the formulation of this group law on the divisor level will be completely sufficient for the question we aim to address due to the uniqueness of the generalized Shioda maps.

We begin by shifting the basis of multi-sections by $k^{m}$-times the $n$-section $s_{m}^{(n)}$ as

$$
\begin{aligned}
& {\left[\tilde{s}_{0}^{(n)}\right]:=\left[s_{0}^{(n)}\right] \oplus k^{m}\left[s_{m}^{(n)}\right],} \\
& {\left[\tilde{s}_{m}^{(n)}\right]:=\left[s_{m}^{(n)}\right] \oplus k^{m}\left[s_{m}^{(n)}\right] .}
\end{aligned}
$$

Using the group law (5.10) and inserting the resulting divisor classes into the generalized Shioda map (5.15) then find that the transformation of $D_{m}^{(n)}$ is given by

$$
D_{m}^{(n)} \mapsto D_{m}^{(n)}-\frac{k^{p}}{n} \pi\left(D_{p}^{(n)} \cdot D_{m}^{(n)}\right),
$$

which differs by a factor of $\frac{1}{n}$ in the vertical part from (4.8a). We emphasize that in this evaluation the ambiguity in the vertical parts is absent after applying the generalized Shioda map.

Let us now analyze the large gauge transformations from a field theory perspective. Recall that in general the actual Kaluza-Klein vector mixes in the Higgsed phase with 
other $\mathrm{U}(1) \mathrm{s}$ as dictated by the zero-multi-section $s_{0}^{(n)}[28,30-33]$. While there are $n-1$ massive U(1)s parameterized by $s_{0}^{(n)}$ only a single U(1) remains massless. To simplify the treatment of the large gauge transformations in such a situation, we again can consider the unHiggsed phase. This will allow us to show that (5.22) is induced by large gauge transformations. In particular we consider the different splits corresponding to the divisor $D_{m}^{(n)}$. Note that $D_{m}^{(n)}$ was obtained in $(5.14)$ by averaging over all divisors $D_{m, l}^{\prime}$, defined in (5.12), which together represent the different choices for the zero-section. Focusing now on a particular divisor $D_{m, l}^{\prime}$ with zero-section $s_{0}^{l}$, we find that the dual gauge field in the unHiggsed phase reads

$$
A^{\prime m, l}=\frac{1}{n}\left(\sum_{i=1}^{n} A_{i}^{m}-\sum_{\substack{i=1 \\ i \neq l}}^{n} A_{i}^{0}\right),
$$

with $A_{i}^{0}, A_{i}^{m}$ dual to $D_{0}^{i}, D_{m}^{i}$. Our main interest is in the form of the large gauge transformation for this vector field $A^{\prime m}$. Therefore let us apply large gauge transformations with winding $k_{i}^{m}$ of the individual constituents $A_{i}^{m, l}$. We find

$$
A_{i}^{m} \mapsto A_{i}^{m}-k_{i}^{m} A_{l}^{0}, \quad \quad A_{i}^{0} \mapsto A_{i}^{0},
$$

where $A_{l}^{0}$ denotes the Kaluza-Klein vector. We conclude that the large gauge transformation acts on $A^{\prime m, l}$ as

$$
A^{\prime m, l} \mapsto A^{\prime m, l}-\frac{\sum_{i=1}^{n} k_{i}^{m}}{n} A_{l}^{0} .
$$

Using the results from section 4 we conclude that the dual divisors transform as

$$
D_{m, l}^{\prime} \mapsto D_{m, l}^{\prime}-\frac{\sum_{i=1}^{n} k_{i}^{p}}{n} \pi\left(D_{p, l}^{\prime} \cdot D_{m, l}^{\prime}\right)
$$

Averaging now as in (5.14) over the different choices for the zero-section we can finally infer that the genuine $\mathrm{U}(1)$ divisors $D_{m}^{(n)}$ in the Higgsed phase transform as

$$
D_{m}^{(n)} \mapsto D_{m}^{(n)}-\frac{\sum_{i=1}^{n} k_{i}^{p}}{n} \pi\left(D_{p}^{(n)} \cdot D_{m}^{(n)}\right) .
$$

This is precisely what we get from (5.22) for appropriate choices of the $k_{i}^{m}$. We finally conclude that shifts in the generalized Mordell-Weil group correspond to Abelian large gauge transformations in the Higgsed phase.

\section{Introducing an arithmetic structure on fibrations with exceptional divisors}

In this section, we focus on elliptic fibrations $\hat{Y}$ with codimension-one singularities leading to non-Abelian gauge groups with matter in F-theory. The resolution of singularities of the elliptic fibration at codimension-one in the base $B$ requires introducing a set of blow-up divisors. In subsection 6.1 we define a novel group action on the set of these blow-up divisors 
in $\hat{Y}$. We are guided by two principles in defining this group structure, one geometric and one field theoretic one.

First, we employ the geometric fact that many geometries $\hat{Y}$ with a Higgsable nonAbelian gauge group can be connected by a number of extremal transitions, corresponding to Higgsing in field theory, to a geometry $\hat{Y}_{\mathrm{H}}$ with a purely Abelian gauge group, i.e. a number of rational sections. Under this transition, the Cartan U(1)s inside the non-Abelian gauge group are mapped to U(1)s associated to the free generators of the Mordell-Weil generators of the Higgsed geometry $\hat{Y}_{\mathrm{H}}$. The postulated group structure on the blow-up divisors of the non-Abelian theory is then nothing but the translational symmetry in the Mordell-Weil group of the Higgsed theory that has been shown to be a geometric symmetry in section 4. In subsection 6.1 we will assume that such a Higgs transition exists and exploit it to define the group structure on $\hat{Y}$. We show this correspondence explicitly in the simplest case of an adjoint Higgsing of $\mathrm{SU}(2)$ to $\mathrm{U}(1)$ in subsection 6.2 and use induction on the number of $\mathrm{U}(1) \mathrm{s}$ to generalize to higher rank groups. Thus, we see that the non-Abelian group structure is required by consistency under motion in the moduli space of F-theory.

Second, we show in subsection 6.1 that in the effective field theory the postulated group action manifests itself simply as non-Abelian large gauge transformations and is therefore trivially a symmetry in an anomaly-free theory. Thus, we claim that the non-Abelian group action should have a direct geometric interpretation on $\hat{Y}$ and does generally exist for any non-Abelian setup, even those lacking Higgsings to Abelian theories.

We note that application of the results from the previous sections implies that the geometric symmetries postulated here imply the cancellation of all pure and mixed gauge anomalies in the effective action of F-theory compactifications on elliptically fibered CalabiYau three- and fourfolds.

\subsection{A group action for exceptional divisors}

As outlined at the beginning of this section, we define in the following a group structure on the set of resolution divisors of codimension-one singularities of an elliptic fibration $\hat{Y}$. We first motivate the group structure geometrically by the connection between Abelian and non-Abelian gauge groups via (un)Higgsing. Then we show that the postulated group law is identified with non-Abelian large gauge transformations, which are automatically a symmetry of the effective theory. Furthermore, we show that the postulated group law leaves key classical intersections on $\hat{Y}$ invariant; in particular the intersections of the transformed exceptional divisors yield the same Cartan matrix as before and the transformed rational sections obey again the defining intersection properties of rational sections discussed in section 3 .

We will start with a purely Abelian theory specified by an elliptic fibration $\hat{Y}_{\mathrm{H}}$ with a Mordell-Weil group generated by elements $s_{m}^{\prime}, m=1, \ldots, n_{\mathrm{U}(1)}$. Although the following arguments hold in general, we will assume that the Mordell-Weil group has no torsion elements. We consider an unHiggsing to a geometry $\hat{Y}$, where a subset of the rational sections are turned into exceptional divisors $D_{I}$ corresponding to a non-Abelian gauge group $G$. As discussed systematically in $[17,27]$, such an unHiggsing is a tuning in the complex structure of $\hat{Y}_{\mathrm{H}}$ so that certain rational sections coincide globally in the tuned 
geometry. Thus, $\hat{Y}$ will have a lower rank Mordell-Weil group with generators denoted by $s_{n}, n=1, \ldots, \tilde{n}_{\mathrm{U}(1)}$ for $\tilde{n}_{\mathrm{U}(1)}<n_{\mathrm{U}(1)}$.

We focus here on the simplest situation possible corresponding to a rank preserving unHiggsing, i.e. a situation with $\operatorname{rk}(G)=n_{\mathrm{U}(1)}-\tilde{n}_{\mathrm{U}(1)}$. Then the non-Abelian gauge theory associated to $\hat{Y}$ is Higgsed back to the original Abelian gauge theory specified by $\hat{Y}_{\mathrm{H}}$ by matter in the adjoint representation. Thus, the divisor groups of $\hat{Y}_{\mathrm{H}}$ and $\hat{Y}$ are of the same dimension and generated by the following elements, respectively:

$$
H_{p}\left(\hat{Y}_{\mathrm{H}}\right)=\left\langle S_{0}^{\prime}, S_{n}^{\prime}, S_{I}^{\prime}, D_{\alpha}^{\prime}\right\rangle, \quad H_{p}(\hat{Y})=\left\langle S_{0}, S_{n}, D_{I}, D_{\alpha}\right\rangle,
$$

where $p=4$ for Calabi-Yau threefolds and $p=6$ for Calabi-Yau fourfolds. Here $S_{0}^{\prime}, S_{n}^{\prime}$ and $S_{I}^{\prime}$ are divisor classes associated to the rational sections on $\hat{Y}_{\mathrm{H}}, S_{0}$ and $S_{n}$ are divisor classes of the sections on $\hat{Y}$. $D_{\alpha}^{\prime}$ and $D_{\alpha}$ are divisors that ascent from divisors in $B$ and define in fact the same classes in $\hat{Y}_{\mathrm{H}}$ and $\hat{Y}$. The index $I=1, \ldots, n_{\mathrm{U}(1)}-\tilde{n}_{\mathrm{U}(1)}$ is the same for both geometries and labels the sections on $\hat{Y}_{\mathrm{H}}$ that are mapped to exceptional divisors $D_{I}$ associated to the group $G$ on $\hat{Y}$.

We propose that the unHiggsing $\hat{Y}_{\mathrm{H}} \rightarrow \hat{Y}$ induces the existence of a map $\varphi$ from the divisor group of $\hat{Y}_{\mathrm{H}}$ to that of $\hat{Y}$,

$$
\varphi: \quad H_{p}\left(\hat{Y}_{\mathrm{H}}\right) \rightarrow H_{p}(\hat{Y}),
$$

with certain properties to be defined next. We we will argue explicitly in subsection 6.2 that the (un)Higgsing processes described in $[5,17,27]$ implies the existence of a map $\varphi$ as described now.

We require $\varphi$ to be a bijective ring homomorphism from the full intersection ring on $\hat{Y}_{\mathrm{H}}$ to that on $\hat{Y}$, i.e. to commute with the intersection pairing of divisors and to be linear. The image of $\varphi$ on the generators of $H_{p}\left(\hat{Y}_{\mathrm{H}}\right)$ with $p=4$ (or $p=6$ for fourfolds) is given by

$$
\varphi\left(S_{0}^{\prime}\right)=S_{0}, \quad \varphi\left(S_{n}^{\prime}\right)=S_{n}, \quad \varphi\left(D_{I}^{\prime}\right)=D_{I}, \quad \varphi\left(D_{\alpha}^{\prime}\right)=D_{\alpha} .
$$

We emphasize that $\varphi$ maps the Shioda map $D_{I}^{\prime}$ of the rational section $s_{I}^{\prime}$ to a Cartan divisor $D_{I}$ of the unHiggsed gauge group $G$ on $\hat{Y}$. Note, however, that (6.3) implies that $\varphi$ does not map the Shioda map $D_{n}^{\prime}$ of a section $s_{n}^{\prime}$ on $\hat{Y}_{\mathrm{H}}$ to the Shioda map $D_{n}$ of $s_{n}$ on $\hat{Y}$. This is clear as the formula for $D_{n}$, according to (3.8), involves the Cartan divisors on $\hat{Y}$ that are absent on $\hat{Y}_{\mathrm{H}}$ and, consequently, do not appear in the formula for $D_{n}^{\prime}$.

We are now in the position to investigate the image of a translation in the Mordell-Weil group of $\hat{Y}_{\mathrm{H}}$ under the map $\varphi$ to the unHiggsed geometry $\hat{Y}$. We are particularly interested in shifts by rational sections $s_{I}^{\prime}$, whose associated Shioda maps $D_{I}^{\prime}$ map to Cartan divisors $D_{I}$ in $\hat{Y}$. To this end we recall the action of a Mordell-Weil translation on $\hat{Y}_{\mathrm{H}}$ on its divisor group. First, we express the Mordell-Weil translations on $\hat{Y}_{\mathrm{H}}$ conveniently in terms of the $D_{I}^{\prime}$. Shifting the Mordell-Weil lattice on $\hat{Y}_{\mathrm{H}}$ by a vector $\oplus k^{I} s_{I}^{\prime}$ we rewrite (4.6) for all sections $s_{\mathcal{M}}^{\prime}:=\left\{s_{0}^{\prime}, s_{m}^{\prime}\right\}$ as

$$
\operatorname{Div}\left(s_{\mathcal{M}}^{\prime} \oplus k^{I} s_{I}^{\prime}\right)=S_{\mathcal{M}}^{\prime}+\sum_{I} k^{I} D_{I}^{\prime}-\frac{1}{2} \sum_{I, J} k^{I} k^{J} \pi\left(D_{I}^{\prime} \cdot D_{J}^{\prime}\right)-\sum_{I} k^{I} \pi\left(S_{\mathcal{M}}^{\prime} \cdot D_{I}^{\prime}\right)
$$


We also recall the general Mordell-Weil group action on a Shioda map $D_{m}^{\prime}$ of a section $s_{m}^{\prime}$ as given in (4.8a). We now perform the unHiggsing by applying the ring homomorphism $\varphi$, employing (6.3), to the formulae in (6.4) and (4.8a). We find the following transformation of divisor classes of sections and Cartan divisors on $\hat{Y}$ by lifting the Mordell-Weil translations on $\hat{Y}_{\mathrm{H}}$ :

$$
\begin{aligned}
& \tilde{S}_{0}=S_{0}+\sum_{I} k^{I} D_{I}-\frac{1}{2} \sum_{I, J} k^{I} k^{J} \pi\left(D_{I} \cdot D_{J}\right) \\
& \tilde{S}_{n}=S_{n}+\sum_{I} k^{I} D_{I}-\frac{1}{2} \sum_{I, J} k^{I} k^{J} \pi\left(D_{I} \cdot D_{J}\right)-\sum_{I} k^{I} \pi\left(S_{n} \cdot D_{I}\right) \\
& \tilde{D}_{I}=D_{I}-\sum_{J} k^{J} \pi\left(D_{J} \cdot D_{I}\right) .
\end{aligned}
$$

We note that the map $\varphi$ simply amounts to $\sum_{I} k^{I} D_{I}^{\prime} \mapsto \sum_{I} k^{I} D_{I}$, as follows from (6.3). Then, we have additionally used $\pi\left(S_{0} \cdot D_{I}\right)=0$ in the first equation since the zero section does not pass through the Cartan divisors on $\hat{Y}$ as expected and $\pi\left(D_{n} \cdot D_{I}\right)=0$ by definition of the Shioda map on the unHiggsed geometry $\hat{Y}$.

From a field theory point of view it is clear that the shifted classes (6.5) correspond to non-Abelian large gauge transformations along the Cartan subalgebra. Indeed one finds that under (6.5) the divisors on $\hat{Y}$ transform as

$$
\left(\begin{array}{c}
\tilde{D}_{0} \\
\tilde{D}_{I} \\
\tilde{D}_{n} \\
\tilde{D}_{\alpha}
\end{array}\right)=\left(\begin{array}{cccc}
1 & k^{J} & 0 & \frac{k^{J} k^{L}}{2} \mathcal{C}_{J L} b^{\beta} \\
0 & \delta_{I}^{J} & 0 & k^{K} \mathcal{C}_{I K} b^{\beta} \\
0 & 0 & \delta_{n}^{k} & 0 \\
0 & 0 & 0 & \delta_{\alpha}^{\beta}
\end{array}\right) \cdot\left(\begin{array}{c}
D_{0} \\
D_{J} \\
D_{k} \\
D_{\beta}
\end{array}\right)
$$

where we have used (3.13) and again recall that $\pi\left(D_{n} \cdot D_{I}\right)=0$. Indeed, (6.6) is precisely the formulae for a non-Abelian large gauge transformation given in (2.15) along the nonAbelian Cartan gauge field $\sum_{J} k_{J} A^{J}$, i.e. for $k^{m}=0$, so that the combination $C_{3}=$ $A^{\Lambda} \wedge\left[D_{\Lambda}\right]$ remains invariant.

In the following we will impose these shifts in F-theory compactifications with nonAbelian gauge symmetry independently of an existing adjoint Higgsing to the maximal torus of $G$. We conclude by showing that the transformed divisor classes (6.5) on $\hat{Y}$ obey the key properties (3.11) and (3.12) of new Cartan divisors and new rational sections, respectively, so that the gauge algebra and the rank of the Mordell-Weil group are invariant. First of all let us note that the $\tilde{S}_{\mathcal{M}}=\left\{\tilde{S}_{0}, \tilde{S}_{n}\right\}$ define good divisor classes for sections. Indeed we find that

$$
\begin{aligned}
\tilde{S}_{0} \cdot \tilde{D}_{I} & =0 \\
\tilde{S}_{\mathcal{M}} \cdot \mathcal{E} & =1 \\
\pi\left(\tilde{S}_{\mathcal{M}} \cdot \tilde{S}_{\mathcal{M}}\right) & =K .
\end{aligned}
$$


Second we check that also the other classical intersection numbers such as (3.13) for the divisors $\tilde{D}_{I}$ are not changed. This indicates that there might exist a new geometric interpretation of the transformed divisors $\left(\tilde{S}_{0}, \tilde{S}_{n}, \tilde{D}_{I}\right)$ as sections and exceptional divisors in an associated geometry. It also hints to the existence of a geometric interpretation for the Mordell-Weil translations lifted from $\hat{Y}_{\mathrm{H}}$ to $\hat{Y}$.

To close this subsection, let us note that we can push the analogy to the elliptic fibration with rational sections even further by defining a so-called zero-node $\Sigma_{0}$ (see also [18]). We introduce $\Sigma_{0}$ as

$$
\Sigma_{0}:=\sum_{I} k^{I} D_{I}
$$

Using this definition the transformations (6.5) can be rewritten in a simpler form eliminating all $k^{I}$-dependence. The freedom to make a shift by a large non-Abelian gauge transformation then translates to 'picking a zero-node'. This is analogous to picking a zero-section in examples with multiple rational sections. For the latter case it has been argued in [18] that this should constitute an actual symmetry of the M-theory to F-theory limit and therefore implies cancellation of Abelian anomalies. For the non-Abelian large gauge transformations and the group action introduced here such a geometric symmetry principle has yet to be established, but would guarantee the cancellation of all non-Abelian anomalies.

\subsection{Arithmetic group structures from Higgs transitions}

Given a gauge theory with non-Abelian gauge group $G$ and matter in the adjoint representation, we can Higgs to $\mathrm{U}(1)^{r}$ with $r=\operatorname{rk}(G)$ by switching on VEVs along the Cartan generators in the adjoint. The inverse process is called unHiggsing a U(1) symmetry. Various examples of unHiggsing U(1) symmetries in F-theory have been considered, see e.g. the most recent works $[5,15,17,27]$ on unHiggsings of up to two $\mathrm{U}(1) \mathrm{s}$. We will employ the unHiggsing of $U(1)$ symmetries to non-Abelian groups in the following in order to provide further geometrical evidence for the existence of the group structure on the exceptional divisors postulated in subsection 6.1. For simplicity, we focus here on the simplest case of the unHiggsing of one $\mathrm{U}(1)$ to $\mathrm{SU}(2)$ as studied in [5, 27]. By induction over the number of $\mathrm{U}(1) \mathrm{s}$, as suggested in [17], the obtained results are expected to generalize to higher rank non-Abelian gauge groups.

It has been shown by Morrison and Park in [5] that the normal form of a general elliptic fibration with a rank one Mordell-Weil group, i.e. a single U(1), is a Calabi-Yau hypersurface $\hat{Y}_{H}$ with elliptic fiber given as the quartic hypersurface in the blow-up of $\mathbb{P}^{2}(1,1,2)$, denoted $\mathrm{Bl}_{1} \mathbb{P}^{2}(1,1,2)$. This space has a toric description. Denoting the projective coordinates on $\mathrm{Bl}_{1} \mathbb{P}^{2}(1,1,2)$ by $[u: v: w: e]$, where $e=0$ is exceptional divisor of the blow-up (with map $[u: v: w: e] \mapsto[u e: v: w e]$ to $\mathbb{P}^{2}(1,1,2)$ ), the elliptic fibration can be brought into the form ${ }^{10}$

$$
e w^{2}+b v^{2} w=u\left(c_{0} u^{3} e^{3}+c_{1} u^{2} e^{2} v+c_{2} u e v^{2}+c_{3} v^{3}\right) .
$$

\footnotetext{
${ }^{10}$ Note that the coefficient of $e w^{2}$ is set to one in order to avoid the $\mathbb{Z}_{2}$ singularity at $u=v=0$, which would give rise to a codimension-one singularity of type $I_{2}$, i.e. an $\mathrm{SU}(2)$ gauge group in F-theory, see $[15,30]$ for an analysis of the geometry with this additional singularity.
} 
The coefficients $c_{i}, i=0,1,2,3$, are sections in specific line bundles that are determined by the the requirement that (6.11) defines a well-defined section of a line bundle on the base $B$ and obeys the Calabi-Yau condition:

\begin{tabular}{c|c} 
section & Class \\
\hline$\left[c_{0}\right]$ & $-4 K-2[b]$ \\
{$\left[c_{1}\right]$} & $-3 K-[b]$ \\
{$\left[c_{2}\right]$} & $-2 K$ \\
{$\left[c_{3}\right]$} & $-K+[b]$ \\
{$[b]$} & {$[b]$}
\end{tabular}

Here, we denote the divisor class of a section by [.] and $-K$ is the anti-canonical divisor of $B$. Note that the class [b] of the divisor $b=0$ is a free parameter of the Calabi-Yau manifold $\hat{Y}$.

The two rational sections of the elliptic fibration are given by

$$
s_{0}^{\prime}: \quad[0: 1: 1:-b], \quad s_{1}^{\prime}: \quad\left[b: 1: c_{3}: 0\right]
$$

where we picked $s_{0}$ as the zero section. ${ }^{11}$ The Shioda map (3.8) of the section $s_{1}^{\prime}$ reads

$$
D_{1}^{\prime}=S_{1}^{\prime}-S_{0}^{\prime}+K-[b]=S_{1}^{\prime}-S_{0}^{\prime}-\left[c_{3}\right]
$$

where we denoted the homology class of the two sections by $S_{0}^{\prime}=\operatorname{Div}\left(s_{0}^{\prime}\right)$ and $S_{1}^{\prime}=\operatorname{Div}\left(s_{1}^{\prime}\right)$.

The divisor $D_{1}^{\prime}$ supports the U(1) of the F-theory compactification on (6.11) as can be seen from the expansion (3.1) of the M-theory three-form. Shifting the origin in the Mordell-Weil lattice, as discussed in subsection 4.2, yields the new divisor classes (4.8a) that were shown to correspond to large Abelian gauge transformations.

The unHiggsing of the $\mathrm{U}(1)$ to an $\mathrm{SU}(2)$ is performed by tuning $b \mapsto 0$ in the elliptic fibration (6.11), as discussed in [5, 27], so that the rational sections in (6.13) coincide globally, except for the locus $c_{3}=0$. As the fiber is toric, this can be achieved by blowing up at $u=e=0$, which amounts to replacing

$$
u \mapsto u e_{1}, \quad e \mapsto e e_{1}
$$

where $e_{1}=0$ is a new exceptional divisor. The hypersurface equation for the unHiggsed geometry $\hat{Y}$ after blow-up reads

$$
e w^{2}=u\left(c_{0} u^{3} e^{3} e_{1}^{6}+c_{1} u^{2} e^{2} e_{1}^{4} v+c_{2} u e e_{1}^{2} v^{2}+c_{3} v^{3}\right) .
$$

The single remaining section on $\hat{Y}$, now denoted by $s_{0}$, is described by $e_{1}=0$ after blow-up, with coordinates $\left[u: v: w: e: e_{1}\right]$ reading

$$
s_{0}: \quad\left[1: 1: 1: c_{3}: 0\right]
$$

\footnotetext{
${ }^{11}$ This convention deviates from the one chosen in [5], but is physically equivalent [18].
} 
showing that $s_{0}$ is holomorphic. We note that the blown-up hypersurface has a Kodaira singularity of type $I_{2}$ at $c_{3}=0$ corresponding to an $\mathrm{SU}(2)$ gauge group in F-theory. Indeed, by setting $c_{3}=0$ in (6.16) we obtain

$$
e\left(w^{2}-u\left(c_{0} u^{3} e^{3} e_{1}^{6}+c_{1} u^{2} e^{2} e_{1}^{4} v+c_{2} u e e_{1}^{2} v^{2}\right)=0,\right.
$$

which describes two $\mathbb{P}^{1}$ 's intersecting at two points. Thus, we identify $S^{\mathrm{SU}(2)}=\left\{c_{3}=0\right\}$ as the divisor supporting the $\mathrm{SU}(2)$ gauge group. As the zero section $s_{0}$ passes through the $\mathbb{P}^{1}$ given by $e=0$, we determine the class of the Cartan divisor $D_{1}$ as

$$
D_{1}=\left[c_{3}\right]-[e]
$$

Furthermore, we see that the divisor $u=0$ does not intersect the hypersurface (6.16), i.e. $\hat{Y} \cap\{u=0\}=0$, due to the Stanley-Reissner ideal of the blown-up ambient space. Using these observations, we infer that the pull-back of the Shioda map (6.14) of the original rational section $s_{1}^{\prime}$ to the unHiggsed geometry $\hat{Y}$ reads

$$
D_{1}^{\prime} \mapsto[e]-\left[c_{3}\right]=-D_{1} .
$$

Clearly, we have $S_{0}^{\prime} \mapsto S_{0}$ while vertical divisor $D_{\alpha}$ map trivially. These are, up to the irrelevant sign in the map of $D_{1}^{\prime}$, precisely the properties of the map $\varphi$ defined in (6.3).

In summary, we see that the Shioda map of the rational sections is mapped, up to sign, to the Cartan divisor of the unHiggsed SU(2) gauge group on $\hat{Y}_{\mathrm{uH}}$. Consequently, the Mordell-Weil shift of a rank one Mordell-Weil group, as introduced in subsection 4.2, is mapped under the transition corresponding to the unHiggsing to $\mathrm{SU}(2)$ to a similar shift of divisors, where the Shioda map is replaced by the Cartan divisor of the $\mathrm{SU}(2)$ (the sign can be absorbed by the integer $k$ in (4.7)). In addition, a similar replacement should apply for unHiggsing a higher rank Mordell-Weil group by induction on its rank, as discussed in [17]. This is expected to establish the existence of the group law postulated in subsection 6.1 on the Cartan divisors of any non-Abelian gauge group in F-theory that can be Higgsed in an adjoint Higgsing to a purely Abelian gauge group. We propose that this group law exists even for those non-Abelian groups that can not be Higgsed, such as the non-Higgsable clusters in [50].

\section{Conclusions}

In this work, we have systematically studied the relationship between the F-theory effective theory on a circle and the geometry of resolved elliptic fibrations. We have developed for the first time the detailed dictionary between large gauge transformations along the circle direction and arithmetic structures as well as geometric symmetries of the elliptic fibration. Our discussion is model-independent, i.e. the structures and correspondences we have uncovered are present in any elliptic or genus-one fibration suitable for F-theory. We summarize the key results of this work and point out some avenues for future works in the following.

We have shown, as suggested in [18], that the translational symmetry in the MordellWeil lattice of rational sections (the so-called translation-by- $Q$ map) corresponds to a 
combination of Abelian and fractional non-Abelian large gauge transformations. This completes the proof of cancellation of all Abelian gauge anomalies in F-theory of [18]. We have explicitly provided the change of all divisor classes under a translation by an arbitrary combination of free generators of the Mordell-Weil group. Most notably, we find that one has to combine the Abelian large gauge transformation with an additional fractional nonAbelian large gauge transformation if there exist fractional charges. Geometrically, this link follows due to fractional contributions to the Shioda map of a rational section, which lead to fractional charges. Field-theoretically, fractional charges are possible in the presence of non-Abelian groups and corresponding Abelian large gauge transformations are then only well-defined if augmented by appropriate fractional non-Abelian ones. We emphasize that the Mordell-Weil translation also acts non-trivially on exceptional divisors. While the geometric explanation of this fact is beyond the scope of this work, we expect a better understanding of the Mordell-Weil group law at singular or resolved fibers at codimensionone to shed light on this issue.

We have also extended the translational symmetry in the Mordell-Weil lattice to shifts by its torsional elements. Similar as before, we have shown that these transformations correspond to special fractional non-Abelian large gauge transformations along the circle. Again, we have presented to action on all divisor classes in the geometry where we stress the non-trivial action on Cartan divisors.

Extending the correspondence between the arithmetic structure of the Mordell-Weil group and symmetries in the effective theories of F-theory, we have found compelling evidence for the existence of a group structure on the set of multi-sections in genus-one fibrations. We refer to this new group as the extended Mordell-Weil group. The study of this novel structure on the divisor level allows us to define a generalized Shioda map for multi-sections, as suggested in [15], which can be used e.g. for the computation of $\mathrm{U}(1)$-charges of matter. The guiding principle in formulating the group law is, given a geometry with an $n$-section, the existence of a different geometry with $n$ sections related by unHiggsing, i.e. a number of conifold transitions. The group law on the multi-sections is then merely a lift of parts of the group law on the sections in the unHiggsed geometry. Using the other results of this work mentioned before, this implies that translations in the extended Mordell-Weil group correspond to Abelian large gauge transformations in the unHiggsed theory. While we have introduced the extended Mordell-Weil group for torus fibrations without rational sections, we speculate that there equally exists an extended group structure for multi-sections in geometries also admitting rational sections.

It is an interesting problem for future works to formulate the postulated group law of the extended Mordell-Weil group in terms of operations on the coordinates of multisections, similar to the usual group law for rational points on elliptic curves. This would reveal the underlying geometric symmetries of genus-one and their associated Jacobian fibrations more directly.

Finally, we have argued for the existence of a novel group structure on the set of exceptional divisors for resolved geometries with non-Abelian gauge groups in F-theory. Here, we have been guided by the effect of non-Abelian large gauge transformations along the circle on the lower-dimensional effective theory on the Coulomb branch. We have 
discovered a group structure and formulated its action on divisor classes of the resolved geometry. The resulting new divisors classes are shown to have all the properties necessary to be interpreted as new exceptional divisors and rational sections. The transformations found are formally completely analogous to those induced by translations in a MordellWeil lattice. Indeed, we argue that this is expected as any non-Abelian gauge theory with adjoint matter can be Higgsed in a rank preserving way to an Abelian theory. We show that under this transition the postulated group law on exceptional divisors is mapped to the group law arising from translations in the Mordell-Weil group of the geometry yielding the Abelian theory. We have demonstrated this explicitly for a single $\mathrm{U}(1)$ described by the geometry in $[5,27]$ and used induction on the number of $\mathrm{U}(1) \mathrm{s}$, following [17], to generalize to higher rank groups. Moreover, as its existence relies just on the existence of non-Abelian large gauge transformations, we expect the postulated group structure on exceptional divisors to always be realized as a geometric symmetry for resolved elliptic fibration with codimension-one singularities.

The explicit construction of the geometric action corresponding to non-Abelian large gauge transformations on the resolved elliptic fibration remains an open problem worth being addressed in future works. We speculate that the relevant geometric operations involve an appropriate combination of base change and quadratic twist. Having an explicit realization of this geometric symmetry action would provide a general proof of cancellation of all mixed and pure gauge anomalies in any given F-theory compactification.

\section{Acknowledgments}

We would like to thank Andreas Braun, Mirjam Cvetic, Antonella Grassi, Jan Keitel, Noppadol Mekareeya, Dave Morrison, Jonas Reuter, Sakura Schäfer-Nameki, and Wati Taylor for illuminating discussions. This work was supported by a grant of the Max Planck Society. It was in part prepared at the Aspen Center for Physics, which is supported by National Science Foundation grant PHY-1066293. D.K. thanks the Max-Planck Institute for Physics in Munich for hospitality during completion of this project.

\section{A Lie theory conventions}

In this appendix we summarize our conventions for the Lie algebra theory used in this work.

We consider a simple Lie algebra $\mathfrak{g}$ associated to the Lie group $G$. The definition of a (preliminary) basis of Cartan generators $\left\{\tilde{T}_{I}\right\}$ with

$$
\operatorname{tr}_{\mathrm{f}}\left(\tilde{T}_{I} \tilde{T}_{J}\right)=\delta_{I J}
$$

will allow us to fix the normalization of the root lattice. The trace $\operatorname{tr}_{\mathrm{f}}$ is taken in the fundamental representation. As in subsection 2.1 we denote the simple roots by $\boldsymbol{\alpha}_{I}, I=$ $1, \ldots, \operatorname{rank} \mathfrak{g}$, the simple coroots are denoted by $\boldsymbol{\alpha}_{I}^{\vee}:=\frac{2 \boldsymbol{\alpha}_{I}}{\left\langle\boldsymbol{\alpha}_{I}, \boldsymbol{\alpha}_{I}\right\rangle}$. 
In order to match with the geometric setup it is important to introduce a coroot-basis $\left\{T_{I}\right\}$ for the Cartan-subalgebra. It is defined by

$$
T_{I}:=\frac{2 \boldsymbol{\alpha}_{I}^{i} \tilde{T}_{i}}{\left\langle\boldsymbol{\alpha}_{I}, \boldsymbol{\alpha}_{I}\right\rangle}
$$

with $\boldsymbol{\alpha}_{I}^{i}$ the components of the simple roots. We furthermore define the (normalized) coroot intersection matrix $\mathcal{C}_{I J}$ as

$$
\mathcal{C}_{I J}=\lambda_{G}^{-1}\left\langle\boldsymbol{\alpha}_{I}^{\vee}, \boldsymbol{\alpha}_{J}^{\vee}\right\rangle
$$

with

$$
2 \lambda_{G}^{-1}=\left\langle\boldsymbol{\alpha}_{\max }, \boldsymbol{\alpha}_{\max }\right\rangle
$$

where $\boldsymbol{\alpha}_{\max }$ is the root of maximal length. The normalization of the Cartan generators $T_{I}$ (in the coroot basis) is then given by

$$
\operatorname{tr}_{\mathrm{f}}\left(T_{I} T_{J}\right)=\lambda_{G} \mathcal{C}_{I J}
$$

Furthermore for some weight $w$ the Dynkin labels are defined as

$$
w_{I}:=\left\langle\boldsymbol{\alpha}_{I}^{\vee}, w\right\rangle .
$$

The Coxeter labels $a^{I}$ denote the components of the highest root $\theta$ in the expansion

$$
\theta=: \sum_{I} a^{I} \boldsymbol{\alpha}_{I} .
$$

Finally in table 1 we display the numbering of the nodes in the Dynkin diagrams, the Coxeter labels and the definition of the fundamental representations of all simple Lie algebras, as well as the values for the normalization factors $\lambda_{G}$ in our conventions.

Open Access. This article is distributed under the terms of the Creative Commons Attribution License (CC-BY 4.0), which permits any use, distribution and reproduction in any medium, provided the original author(s) and source are credited.

\section{References}

[1] C. Vafa, Evidence for F-theory, Nucl. Phys. B 469 (1996) 403 [hep-th/9602022] [inSPIRE].

[2] D.R. Morrison and C. Vafa, Compactifications of F-theory on Calabi-Yau threefolds (II), Nucl. Phys. B 476 (1996) 437 [hep-th/9603161] [INSPIRE].

[3] T.W. Grimm and T. Weigand, On Abelian Gauge Symmetries and Proton Decay in Global F-theory GUTs, Phys. Rev. D 82 (2010) 086009 [arXiv: 1006.0226] [INSPIRE].

[4] D.S. Park, Anomaly Equations and Intersection Theory, JHEP 01 (2012) 093 [arXiv:1111.2351] [INSPIRE].

[5] D.R. Morrison and D.S. Park, F-Theory and the Mordell-Weil Group of Elliptically-Fibered Calabi-Yau Threefolds, JHEP 10 (2012) 128 [arXiv:1208.2695] [INSPIRE]. 
[6] V. Braun, T.W. Grimm and J. Keitel, New Global F-theory GUTs with U(1) symmetries, JHEP 09 (2013) 154 [arXiv: 1302.1854] [INSPIRE].

[7] J. Borchmann, C. Mayrhofer, E. Palti and T. Weigand, Elliptic fibrations for $\mathrm{SU}(5) \times \mathrm{U}(1) \times \mathrm{U}(1)$ F-theory vacua, Phys. Rev. D 88 (2013) 046005 [arXiv:1303. 5054] [INSPIRE].

[8] M. Cvetič, D. Klevers and H. Piragua, F-Theory Compactifications with Multiple U(1)-Factors: Constructing Elliptic Fibrations with Rational Sections, JHEP 06 (2013) 067 [arXiv: 1303.6970] [INSPIRE].

[9] T.W. Grimm, A. Kapfer and J. Keitel, Effective action of 6D F-theory with U(1) factors: Rational sections make Chern-Simons terms jump, JHEP 07 (2013) 115 [arXiv:1305.1929] [INSPIRE].

[10] V. Braun, T.W. Grimm and J. Keitel, Geometric Engineering in Toric F-theory and GUTs with U(1) Gauge Factors, JHEP 12 (2013) 069 [arXiv:1306.0577] [INSPIRE].

[11] M. Cvetič, A. Grassi, D. Klevers and H. Piragua, Chiral Four-Dimensional F-theory Compactifications With SU(5) and Multiple U(1)-Factors, JHEP 04 (2014) 010 [arXiv:1306.3987] [INSPIRE].

[12] J. Borchmann, C. Mayrhofer, E. Palti and T. Weigand, SU(5) Tops with Multiple U(1)s in F-theory, Nucl. Phys. B 882 (2014) 1 [arXiv:1307.2902] [inSPIRE].

[13] M. Cvetič, D. Klevers and H. Piragua, F-Theory Compactifications with Multiple U(1)-Factors: addendum, JHEP 12 (2013) 056 [arXiv: 1307.6425] [INSPIRE].

[14] M. Cvetič, D. Klevers, H. Piragua and P. Song, Elliptic fibrations with rank three Mordell-Weil group: F-theory with $\mathrm{U}(1) \times \mathrm{U}(1) \times \mathrm{U}(1)$ gauge symmetry, JHEP $03(2014) 021$ [arXiv: 1310.0463] [INSPIRE].

[15] D. Klevers, D.K. Mayorga Pena, P.-K. Oehlmann, H. Piragua and J. Reuter, F-Theory on all Toric Hypersurface Fibrations and its Higgs Branches, JHEP 01 (2015) 142 [arXiv: 1408.4808] [INSPIRE].

[16] C. Lawrie, S. Schäfer-Nameki and J.-M. Wong, F-theory and All Things Rational: Surveying U(1) Symmetries with Rational Sections, JHEP 09 (2015) 144 [arXiv:1504.05593] [INSPIRE].

[17] M. Cvetič, D. Klevers, H. Piragua and W. Taylor, General U(1) $\times$ U(1) F-theory compactifications and beyond: geometry of unHiggsings and novel matter structure, JHEP 11 (2015) 204 [arXiv: 1507.05954] [INSPIRE].

[18] T.W. Grimm and A. Kapfer, Anomaly Cancelation in Field Theory and F-theory on a Circle, arXiv: 1502.05398 [INSPIRE].

[19] T.W. Grimm and H. Hayashi, F-theory fluxes, Chirality and Chern-Simons theories, JHEP 03 (2012) 027 [arXiv:1111.1232] [INSPIRE].

[20] M. Cvetič, T.W. Grimm and D. Klevers, Anomaly Cancellation And Abelian Gauge Symmetries In F-theory, JHEP 02 (2013) 101 [arXiv: 1210.6034] [INSPIRE].

[21] H. Hayashi, C. Lawrie, D.R. Morrison and S. Schäfer-Nameki, Box Graphs and Singular Fibers, JHEP 05 (2014) 048 [arXiv: 1402.2653] [INSPIRE].

[22] A.P. Braun and S. Schäfer-Nameki, Box Graphs and Resolutions I, Nucl. Phys. B 905 (2016) 447 [arXiv: 1407.3520] [INSPIRE]. 
[23] M. Esole, S.-H. Shao and S.-T. Yau, Singularities and Gauge Theory Phases, Adv. Theor. Math. Phys. 19 (2015) 1183 [arXiv:1402.6331] [InSPIRE].

[24] M. Esole, S.-H. Shao and S.-T. Yau, Singularities and Gauge Theory Phases II, arXiv: 1407.1867 [INSPIRE].

[25] J. de Boer et al., Triples, fluxes and strings, Adv. Theor. Math. Phys. 4 (2002) 995 [hep-th/0103170] [INSPIRE].

[26] V. Braun and D.R. Morrison, F-theory on Genus-One Fibrations, JHEP 08 (2014) 132 [arXiv: 1401.7844] [INSPIRE].

[27] D.R. Morrison and W. Taylor, Sections, multisections and U(1) fields in F-theory, arXiv: 1404.1527 [INSPIRE].

[28] M. Cvetič, R. Donagi, D. Klevers, H. Piragua and M. Poretschkin, F-theory vacua with $\mathbb{Z}_{3}$ gauge symmetry, Nucl. Phys. B 898 (2015) 736 [arXiv:1502.06953] [INSPIRE].

[29] V. Braun, T.W. Grimm and J. Keitel, Complete Intersection Fibers in F-theory, JHEP 03 (2015) 125 [arXiv: 1411.2615] [INSPIRE].

[30] L.B. Anderson, I. García-Etxebarria, T.W. Grimm and J. Keitel, Physics of F-theory compactifications without section, JHEP 12 (2014) 156 [arXiv:1406.5180] [INSPIRE].

[31] I. García-Etxebarria, T.W. Grimm and J. Keitel, Yukawas and discrete symmetries in F-theory compactifications without section, JHEP 11 (2014) 125 [arXiv:1408.6448] [INSPIRE].

[32] C. Mayrhofer, E. Palti, O. Till and T. Weigand, Discrete Gauge Symmetries by Higgsing in four-dimensional F-theory Compactifications, JHEP 12 (2014) 068 [arXiv:1408.6831] [INSPIRE].

[33] C. Mayrhofer, E. Palti, O. Till and T. Weigand, On Discrete Symmetries and Torsion Homology in F-theory, JHEP 06 (2015) 029 [arXiv: 1410.7814] [INSPIRE].

[34] P.K. Townsend, K. Pilch and P. van Nieuwenhuizen, Selfduality in Odd Dimensions, Phys. Lett. B 136 (1984) 38 [Addendum ibid. B 136 (1984) 443] [INSPIRE].

[35] F. Bonetti, T.W. Grimm and S. Hohenegger, A Kaluza-Klein inspired action for chiral p-forms and their anomalies, Phys. Lett. B 720 (2013) 424 [arXiv:1206.1600] [INSPIRE].

[36] F. Bonetti and T.W. Grimm, Six-dimensional $(1,0)$ effective action of F-theory via M-theory on Calabi-Yau threefolds, JHEP 05 (2012) 019 [arXiv:1112.1082] [INSPIRE].

[37] M.B. Green and J.H. Schwarz, Anomaly Cancellation in Supersymmetric D = 10 Gauge Theory and Superstring Theory, Phys. Lett. B 149 (1984) 117 [InSPIRE].

[38] A. Sagnotti, A Note on the Green-Schwarz mechanism in open string theories, Phys. Lett. B 294 (1992) 196 [hep-th/9210127] [INSPIRE].

[39] V. Sadov, Generalized Green-Schwarz mechanism in F-theory, Phys. Lett. B 388 (1996) 45 [hep-th/9606008] [INSPIRE].

[40] T.W. Grimm, The $N=1$ effective action of F-theory compactifications, Nucl. Phys. B $\mathbf{8 4 5}$ (2011) 48 [arXiv: 1008.4133] [INSPIRE].

[41] J.A. Harvey, Magnetic monopoles, duality and supersymmetry, in proceedings of the Theoretical Advanced Study Institute in Elementary Particle Physics (TASI 96): Fields, Strings, and Duality, Boulder, Colorado U.S.A., 2-28 June (1996). hep-th/9603086 [INSPIRE]. 
[42] T. Banks and N. Seiberg, Symmetries and Strings in Field Theory and Gravity, Phys. Rev. D 83 (2011) 084019 [arXiv:1011.5120] [InSPIRE].

[43] S. Hellerman and E. Sharpe, Sums over topological sectors and quantization of Fayet-Iliopoulos parameters, Adv. Theor. Math. Phys. 15 (2011) 1141 [arXiv:1012.5999] [INSPIRE].

[44] D.R. Morrison and C. Vafa, Compactifications of F-theory on Calabi-Yau threefolds. (I), Nucl. Phys. B 473 (1996) 74 [hep-th/9602114] [InSPIRE].

[45] S. Ferrara, R. Minasian and A. Sagnotti, Low-energy analysis of $M$ - and F-theories on Calabi-Yau threefolds, Nucl. Phys. B 474 (1996) 323 [hep-th/9604097] [INSPIRE].

[46] F. Denef, Les Houches Lectures on Constructing String Vacua, arXiv:0803.1194 [INSPIRE].

[47] C. Mayrhofer, D.R. Morrison, O. Till and T. Weigand, Mordell-Weil Torsion and the Global Structure of Gauge Groups in F-theory, JHEP 10 (2014) 16 [arXiv:1405.3656] [InSPIRE].

[48] J.H. Silverman, The arithmetic of elliptic curves, in Graduate Texts in Mathematics, volume 106, Springer-Verlag, New York U.S.A. (2009).

[49] P.S. Aspinwall and D.R. Morrison, Nonsimply connected gauge groups and rational points on elliptic curves, JHEP 07 (1998) 012 [hep-th/9805206] [INSPIRE].

[50] D.R. Morrison and W. Taylor, Classifying bases for $6 D$ F-theory models, Central Eur. J. Phys. 10 (2012) 1072 [arXiv: 1201.1943] [INSPIRE]. 